\title{
8. FREQUENCY AND SEQUENCY ANALYSES OF PETROPHYSICAL LOG DATA AND INDUCTIVELY COUPLED ARGON PLASMA ANALYSIS OF SEDIMENTS IN THE LAU BASIN ${ }^{1}$
}

\author{
Cedric M. Griffiths, ${ }^{2}$ Reidulv Bøe, ${ }^{3}$ and Richard A. Hodkinson ${ }^{4}$
}

\begin{abstract}
The results of inductively coupled argon plasma (ICAP) chemical analyses carried out on some 300 core samples from Ocean Drilling Program Sites 834, 835, 838, and 839 are presented. These sites were drilled during Leg 135 in the Lau Basin.

The data are compared with total gamma (SGR) wireline logs at Sites 834 and 835. Pliocene (Piacenzian) nannofossil Zone $\mathrm{CN} 12$, which has been identified at Sites 834 and 835 , is examined in detail using spectral analyses on core and wireline logs.

The potassium and calcium concentrations from the core material were used to calculate an objective depth-to-geological time stretching function, which improved the stratigraphic correlation between sites. The integrated use of chemical analyses, wireline-log data and paleomagnetic results improved confidence in the correlations obtained. Although no significant sedimentation periodicities were obtained from the two sites, a common concentration of energy between 30 and $60 \mathrm{k} . \mathrm{y}$. was recorded.
\end{abstract}

\section{INTRODUCTION}

The Tonga Arc system is divisible into three morphotectonic elements (Karig, 1970; Malahoff et al., 1982) (Fig. 1A). Extending from the Tonga Trench, these are the Tonga Ridge, constituting the forearc (Tonga Platform) and active arc (Tofua Arc); the Lau Basin, representing the actively spreading backarc basin; and the Lau Ridge, forming the remnant arc.

It has been shown that rifting of the Lau-Tonga Ridge and the formation of the Lau Basin was initiated approximately $6 \mathrm{Ma}$ ago (Parson, Hawkins, Allan, et al., 1992); however, organized seafloor spreading began only within the last 1-2 m.y. The Western Lau Basin is characterized by a number of small, fault-bounded, north-trending, partly sedimented sub-basins. Sites 834 and 835 lie in sub-basins between 100 and $200 \mathrm{~km}$ east of the axis of the Lau Ridge, whereas Sites 838 and 839 lie in sub-basins much closer to the Eastern Lau Spreading Center (Fig. 1A). The oldest known sediments in the Lau Basin (5.6 Ma old) occur at Site 834. Figure 1B shows the sections analyzed and the stratigraphy.

Because of the lack of overlap between the stratigraphy at all sites, only two of the four available sites were chosen for the present analysis. Pliocene (Piacenzian) calcareous nannofossil Zone CN12 was cored continuously at Sites 834 and 835 where the Gauss paleomagnetic reversals Mammoth (3.15 Ma, 65 and $149 \mathrm{mbsf}$, respectively) and Kaena (3.01 Ma, 61 and $137.5 \mathrm{mbsf}$, respectively), and the Gauss/Matuyama boundary ( $2.48 \mathrm{Ma}, 46$ and $95 \mathrm{mbsf}$, respectively) were also identified (Parson, Hawkins, Allan, et al., 1992). The error on the isotopic dating of these chron boundaries is probably around \pm 0.1 m.y. (Harland et al., 1989). Core recovery was $100 \%$ over this interval at both sites, and both the paleomagnetic data and biostratigraphy are well matched. We have, therefore, a very good chance at these sites of making a reasonable conversion between depth and geological time over this restricted interval. The depths involved are from 46 to $65 \mathrm{mbsf}$ at Site 834, and from 95 to 149 mbsf at Site 835 .

The aim of this work was to examine sediment cyclicity in a narrow Pliocene window at high resolution. Calculated average (com-

\footnotetext{
${ }^{1}$ Hawkins, J., Parson, L., Allan, J., et al., 1994. Proc. ODP, Sci. Results, 135: College Station, TX (Ocean Drilling Program).

${ }^{2}$ BP/Statoil Research Collaboration, Ranheimsveien 10, N-7004 Trondheim, Norway.

${ }^{3}$ Geological Survey of Norway, P.O. Box 3006 - Lade, N-7002 Trondheim, Norway

${ }^{4}$ Department of Geology, Imperial College of Science, Technology and Medicine. London SW7 2BP, United Kingdom.
}

pacted) sedimentation rates within the Piacenzian at the two sites (Table 1) vary from $28 \mathrm{~m} / \mathrm{m} . y .(2.8 \mathrm{~cm} / \mathrm{k} . \mathrm{y}$.) at Site 834 to $80 \mathrm{~m} / \mathrm{m}$.y. $(8 \mathrm{~cm} / \mathrm{k} . \mathrm{y}$. $)$ at Site 835 , assuming that the magnetic reversal ages are accurate. The use of compacted rather than decompacted sedimentation rates is not of importance here because: (1) the porosity trend over the sections of interest are not significant; (2) decompaction algorithms for volcaniclastic sediments are not yet widely accepted and may introduce more errors; and (3) the spectral analyses are conducted in time, not depth, and the small differences in compaction between magnetic reversal tie points are insignificant.

The ability to identify sediment cyclicity of a given frequency in depth or time depends on the sample interval used. Worthington (1990) has discussed the relationship between wireline-log measurement sensitivity and resolving power for common logging tools. We have chosen to use the gamma log because of the sensitivity of the gamma tool to ${ }^{40} \mathrm{~K}$, the availability of potassium measurements from two independent sources for these holes, and the relationship between $\mathrm{K}$-rich volcanic sands and $\mathrm{K}$-poor nannofossil ooze that is potentially cyclic in nature. In choosing the gamma-log response, we have a tool with a 0.3 - to 0.4 -m sensitivity (minimum half-cycle length resolvable by the tool (Worthington, 1990, p. 126). This implies that in the Piacenzian at Site 834 the gamma log could resolve periods of greater than 28 k.y. $(2 \times 400 \mathrm{~m} / 28 \mathrm{~m} / \mathrm{m} . \mathrm{y}$.), whereas at Site 835 the minimum resolvable period would be $10 \mathrm{k} . \mathrm{y}$. $(2 \times 400 \mathrm{~m} / 80 \mathrm{~m} / \mathrm{m}$.y. $)$. The gamma response was evenly sampled at $0.1524 \mathrm{~m}$ (6 in.). Small variations caused by cable stretch may be expected but the cable tension on these runs did not indicate any serious variability.

The cores were sampled at irregular intervals, as shown in Figures $2 \mathrm{~A}$ and $2 \mathrm{~B}$. Ninety-five percent of the samples analyzed for potassium by Hodkinson (hereafter identified as the HK data set) have a sample interval of $<1.8 \mathrm{~m}$, with a median value of $0.33 \mathrm{~m}$ (Hole $834 \mathrm{~A}$ ) and $0.5 \mathrm{~m}$ (Hole $835 \mathrm{~A}$ ). Of the samples analyzed by Bøe for 29 elements (hereafter identified as the ICAP data set), the median sample intervals were $0.7 \mathrm{~m}$ (Hole $834 \mathrm{~A})$ and $1.0 \mathrm{~m}$ (Hole $835 \mathrm{~A})$, with $95 \%$ of the intervals being under $2 \mathrm{~m}$.

The raw Nyquist (minimum resolvable) wavelength using core samples would be between 1.4 and $4 \mathrm{~m}$. Translating these depth intervals to time (using the average sedimentation rates) produced minimum resolvable periods of 50-143 k.y. at Site 834, and 18-50 k.y. at Site 835, given the age-to-depth assumptions discussed above. While the sampling intervals were too great to be useful for spectral analysis over the dated time interval, the chemical data has been invaluable in calculating the nonlinear sedimentation rates and are thus an integral part of the spectral analysis. 

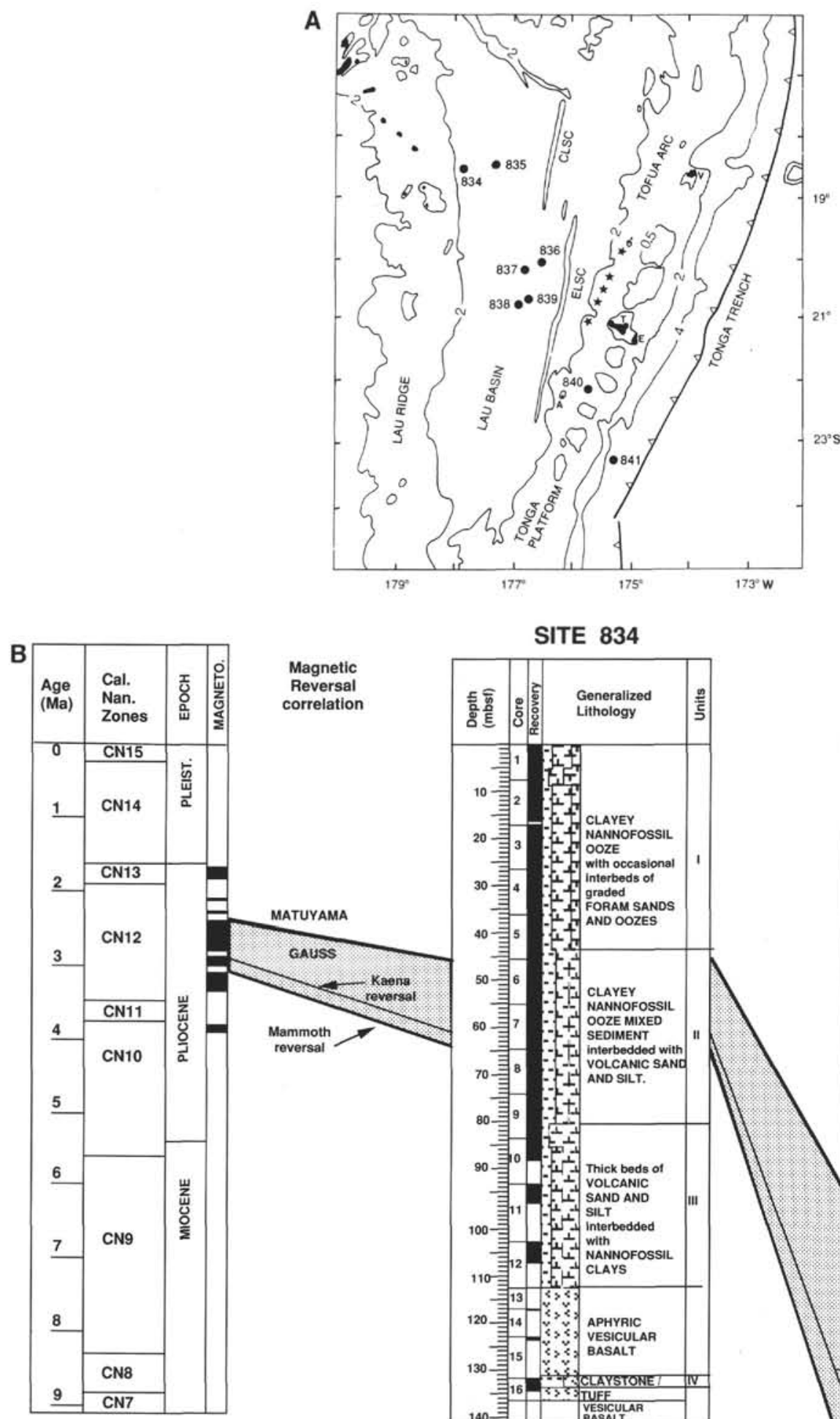

SITE 834

SITE 835

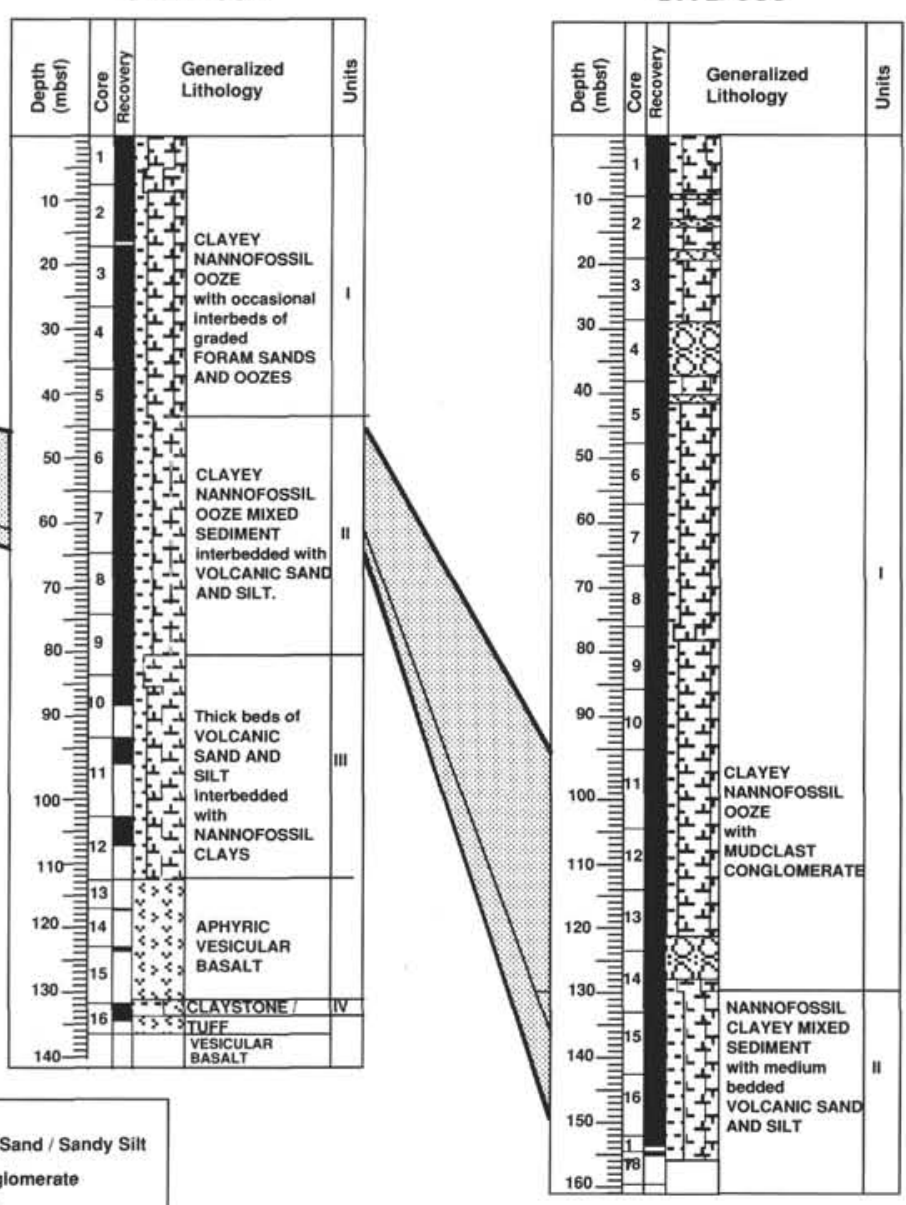

E Foraminiferal Ooze $\square$ Silty Sand / Sandy Sitt

- Nannofossil Ooze $\mathrm{Q}$ Congiomerate

日 Clay/Claystone $Q$ Tutt

Figure 1. A. Regional setting for the Leg 135 drill sites showing the major geological features of the Lau Basin and Tonga Platform. Contour interval in kilometers. Islands abbreviations are $\mathrm{T}=\mathrm{T}$ Tongatapu, $\mathrm{E}={ }^{\prime} \mathrm{Eua}, \mathrm{V}=\mathrm{Vavau}$, and $\mathrm{A}=\mathrm{Ata}$. The locations of the Central Lau (CLSC) and Eastern Lau spreading centers (ELSC) are shown. Stars indicate some of the active volcanoes of the Tofua Arc. B. Stratigraphy of Sites 834 and 835 , Leg 135. The sections of interest as defined by magnetic reversal stratigraphy are shaded. 
Table 1. Statistics for data set 1 at Sites $\mathbf{8 3 4}, \mathbf{8 3 5}, \mathbf{8 3 8}$, and 839.

\begin{tabular}{|c|c|c|c|c|}
\hline Hole & $\begin{array}{l}\text { Depth } \\
\text { (mbsf) }\end{array}$ & $\begin{array}{l}\text { Age } \\
(\mathrm{Ma})\end{array}$ & $\begin{array}{l}\text { Number } \\
\text { of samples }\end{array}$ & $\begin{array}{l}\text { Sedimentation rates } \\
(\mathrm{mm} / \mathrm{k} . \mathrm{y} .)\end{array}$ \\
\hline $834 \mathrm{~A}$ & $27.57-104.80$ & $1.3-3.9$ & 84 & $\begin{array}{l}13(27.57-61 \mathrm{mbsf}) \\
158(61-94 \mathrm{mbsf}) \\
45(94-104.80 \mathrm{mbsf})\end{array}$ \\
\hline $835 \mathrm{~A}$ & $40.52-153.43$ & $0.4-3.1$ & 117 & $\begin{array}{l}92 \text { ( } 40.52-55 \mathrm{mbsf}) \\
15 \text { (55-86 mbsf) } \\
120(86-153.43 \mathrm{mbsf})\end{array}$ \\
\hline $838 \mathrm{~A}$ & $32.60-97.06$ & $1.0-1.9$ & 37 & $\begin{array}{l}40(32.60-55 \mathrm{mbsf}) \\
177(55-97.06 \mathrm{mbsf})\end{array}$ \\
\hline $839 \mathrm{~A}$ & $42.90-99.55$ & $1.0-1.7$ & 40 & $\begin{array}{l}17(42.90-56 \mathrm{mbsf}) \\
882(56-99.55 \mathrm{mbsf})\end{array}$ \\
\hline
\end{tabular}

Note: Biostratigraphic ages and sedimentation rates are from Nishi and Chaproniere (this volume)

\section{LITHOSTRATIGRAPHIC SUMMARY}

The lithostratigraphy at Sites $834,835,838$, and 839 has been described in Parson, Hawkins, Allan, et al. (1992). We will give only a short summary of the lithostratigraphy of the two sequences studied in this project (Fig. 1B).

At Site 834 the sediments of Unit I $(0-42 \mathrm{~m}$ below seafloor, or $\mathrm{mbsf}$ ) consist of clayey nannofossil oozes with some interbeds of foraminifer sand and foraminifer ooze. Unit II (42-78 mbsf) consists of clayey nannofossil mixed sediments interbedded with volcanic sands and silts, whereas Unit III (78-112.5 mbsf) is made up of thick-bedded volcanic sands and silts interbedded with nannofossil clayey mixed sediments.

At Site 835 , Unit I ( $0-130$ mbsf) consists of clayey nannofossil oozes and mudclast conglomerates, with interbeds of foraminifer sand and volcanic silt. According to Rothwell et al. (this volume), most of the unit is made up of debrites, rafted blocks, and thick turbidite muds with basal foraminifer sands. Only $33-34 \mathrm{~m}$ of the unit consists of pelagite. Unit II (130-155.5 mbsf) is composed of nannofossil clayey mixed sediments with interbeds of volcanic sand and volcanic silt.

The majority of the volcaniclastic deposits at Sites 834 and 835 are interpreted as turbidites, although thin pyroclastic fallout ash layers also occur.

All the recovered pelagic clayey nannofossil oozes at the backarc sites are stained a distinctive reddish brown color by hydrothermally derived, iron oxyhydroxides, suggesting that hydrothermal activity has been ongoing throughout the history of the Lau Basin.

\section{INDUCTIVELY COUPLED ARGON PLASMA ATOMIC EMISSION SPECTROSCOPY}

Two different geochemical data sets were available. The ICAP data set consists of geochemical data from analyses of 278 samples of poorly consolidated pelagic and volcaniclastic sediments. These were analyzed for 29 elements (Table 2) by ICAP atomic emission spectroscopy. All the samples were prepared and analyzed according to a standard procedure (Ødegård, 1981, 1983). Some of the samples were taken as physical properties (PP) samples. In the shipboard analyses these were heated to $110^{\circ} \mathrm{C}$ for $24 \mathrm{hr}$, but one assumes that this had little effect on the concentration of the elements analyzed. Some of the samples were ground in an agate mortar or in an agate Tema mill before preparing the sample according to the methods described below. All concentrations are based on dry sample weights.

The HK data set consists of potassium values from Hodkinson and Cronan (this volume). Many of the analyses within the two data sets are on parallel samples. The difference in chemical composition between these parallel samples is mainly a result of differences in the sample dissolution procedures.

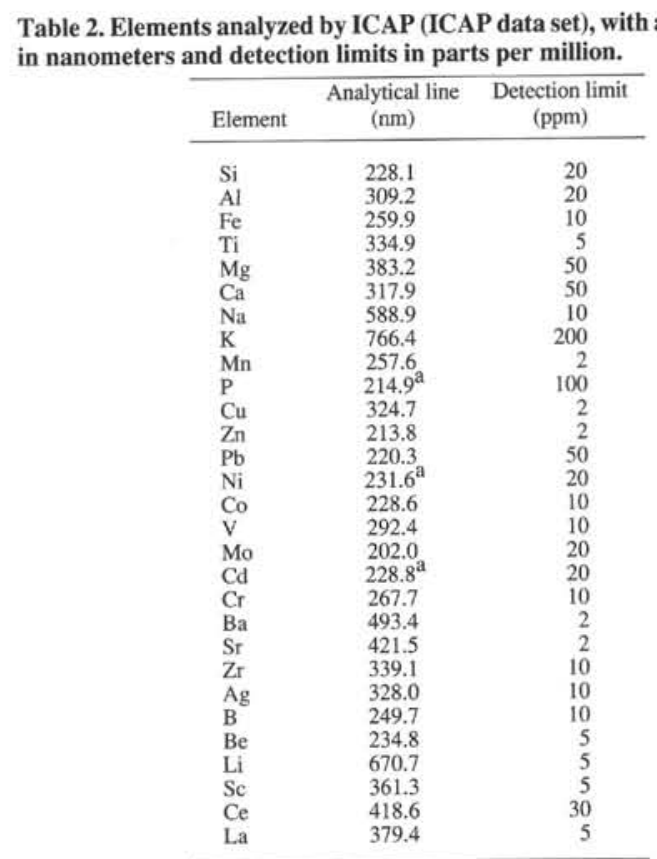

Notes: Analytical lines are given in nanometers, and detection limits in parts per million. (a) $=2$ nd order correlations.

\section{Sample Preparation and Analytical Method (ICAP)}

The samples were homogenized in the plastic bags. Subsamples were then dried for $48 \mathrm{hr}$ at $50^{\circ} \mathrm{C}$ and rehomogenized with a glass rod.

Elements were extracted according to the Norwegian Standard (NS 4770), in which $1 \mathrm{~g}$ of dried material is extracted in $20 \mathrm{~mL} 7 \mathrm{~N}$ $\mathrm{HNO}_{3}$ in sealed borosilicate bottles in an autoclave at $120^{\circ} \mathrm{C}$ for 30 min. After cooling the solutions were filtered through Selecta folding filters No. 595 and transferred to plastic bottles.

The elements were analyzed with an inductively coupled plasma simultaneous spectrometer (Jarrel Ash, Model 975 ICAPAtomComp) with a pneumatic Jarrel Ash cross-flow nebulizer. Constant liquid flow was obtained by a peristaltic pump. All gas flows were controlled by electronic mass flow controllers for maximum stability.

The RF generator was a Plasma Term HFP 2000 D with crystal controlled frequency $(27.12 \mathrm{MHz})$. The supplied effect was $1.1 \mathrm{~kW}$, and the sample uptake was $1.2 \mathrm{~mL} / \mathrm{min}$. The analytical lines used for the various elements are given in Table 2.

The present sediment samples proved to contain large amounts of $\mathrm{Ca}$, and, to bring the matrix element concentration in the final analysis solutions down to an acceptable level, it was necessary to dilute the extracts 1:9 with water before the standard 1:4 dilution with internal reference element solution. This gives a dilution factor of 1000 , which is a factor of 10 higher than that used by the standard procedure for geological materials. Detection limits are, as a result of this, a factor of 10 higher than by normal procedures (Table 2).

Yttrium, in a concentration of $16 \mu \mathrm{M} / \mathrm{mL}$, was used as a reference element for all elements, except the alkalis $\mathrm{Na}, \mathrm{K}$, and Li. Synthetically prepared standards were used to calibrate the instrument, and background correction was used for most of the trace elements. The analytical results are given in Tables 3-6.

\section{Sample Preparation and Analytical Method (HK)}

All samples were air dried at room temperature over silica gel and finely ground in agate vessels. Subsequent bulk chemical analyses were performed by inductively coupled plasma atomic emission spectroscopy (ICAPAES) after total digestion with a mixture of nitric, perchloric, and hydrofluoric acids and subsequent leaching with $1 \mathrm{M}$ 
Table 3. Average bulk composition in percentage of sediment samples from Holes 834A in the Lau Basin (ICAP data set).

\begin{tabular}{|c|c|c|c|c|c|c|c|c|c|c|c|c|c|c|c|c|c|c|c|c|c|c|c|}
\hline & $\ldots$ & & & & & & & & & & & & & & & & & & & & $B$ & LI & La \\
\hline$\frac{1}{2}$ & \begin{tabular}{l|}
27.570 \\
28.450
\end{tabular} & & $\begin{array}{l}0.002 \\
0.0036\end{array}$ & $\begin{array}{r}1.7 \\
0.38 \\
\end{array}$ & \begin{tabular}{l|}
2.31 \\
0.37 \\
\end{tabular} & \begin{tabular}{|c|}
0.0751 \\
0.0215
\end{tabular} & \begin{tabular}{|l|}
0.73 \\
1.14
\end{tabular} & \begin{tabular}{|l|}
27.31 \\
34.48 \\
\end{tabular} & \begin{tabular}{c|}
1.69 \\
0.8
\end{tabular} & \begin{tabular}{|c|}
0.45 \\
0.496 \\
\end{tabular} & \begin{tabular}{l|}
0.97 \\
0.25
\end{tabular} & \begin{tabular}{r|}
0.1 \\
0.0515
\end{tabular} & 0.0107 & 0.005 & 0.0034 & 0.002 & 0.007 & 0.0346 & 0.12 & 0.0014 & \begin{tabular}{|c|c|c|c|} 
\\
\end{tabular} & 0.002 & 0.0012 \\
\hline & $\frac{2.490}{29.100}$ & & 0.0053 & \begin{tabular}{r|r|}
0.38 \\
1.79
\end{tabular} & $\mid$\begin{tabular}{|l|}
243 \\
\end{tabular} & $\begin{array}{l}0.0979 \\
0.079\end{array}$ & 0.82 & \begin{tabular}{|l|}
27.06 \\
\end{tabular} & 1.62 & \begin{tabular}{|l|}
0.006 \\
0.29 \\
\end{tabular} & \begin{tabular}{l|}
.25 \\
0.82
\end{tabular} & 年 0.0675 & 0.011 & $\frac{0.00644}{0.0064}$ & $\mid \begin{array}{c}-0.002 \\
0.0043\end{array}$ & - & $\frac{0.0015}{0.0077}$ & $\frac{0.0048}{0.0415}$ & $\frac{0.21}{0.12}$ & 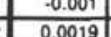 & -0.001 & 0.0018 & \\
\hline & & 41 & 0.0067 & 1.22 & 2.55 & 0.0743 & 0.54 & \begin{tabular}{|l|}
25.82 \\
\end{tabular} & 1.6 & 0.27 & 1.11 & 0.12 & & & & & & & & & & & 0.00017 \\
\hline & 30.600 & 163 & 0.002 & 1.64 & 2.56 & 0.0776 & 0.69 & \begin{tabular}{|l|}
27.24 \\
\end{tabular} & 1.65 & 0.47 & 0.94 & 0.11 & .0107 & .0054 & $\begin{array}{c}0.0056 \\
\end{array}$ & $\overline{j 18}$ & 0087 & $\sqrt{.0503}$ & & $\overline{018}$ & 0.0036 & $\sqrt{.0018}$ & \\
\hline & & 165 & 0.002 & 1.35 & 2.5 & 0.071 & 0.61 & \begin{tabular}{|l|}
26.89 \\
\end{tabular} & 1.33 & 0.41 & 1.16 & 0.1 & .0107 & 0.0056 & 0.0055 & .0019 & 0.0093 & 0.0431 & 0.14 & .0016 & $\overline{0.0027}$ & .0015 & .0012 \\
\hline & 31.500 & 164 & 0.002 & 1.62 & 2.26 & 0.0809 & 072 & \begin{tabular}{|l|}
24.68 \\
\end{tabular} & 1.63 & 0.44 & 0.88 & 0.077 & 0119 & 501 & , & sover & 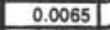 & 80218 & & & & & \\
\hline 8 & \begin{tabular}{|c|}
31.830 \\
\end{tabular} & 15 & $\begin{array}{l}.0 .002 \\
\end{array}$ & 1.55 & 2.41 & 0.0875 & 0.7 & \begin{tabular}{|l|}
27.84 \\
\end{tabular} & 1.6 & \begin{tabular}{l|l}
0.32 \\
\end{tabular} & 1.13 & 0.0985 & 0.0116 & 0.007 & 0.0066 & $\begin{array}{l}0.0022 \\
\end{array}$ & $\mid 0.0088$ & 0.0416 & 0.13 & $\overline{0018}$ & 0.0013 & 0.0016 & 0014 \\
\hline & $31.830 \mid$ & 18 & 0.0048 & 1.06 & 0.94 & 0.0687 & 0.53 & 2.93 & 1.11 & 0.23 & 0.2 & 0.0358 & 0.0058 & 0.0034 & 0.001 & 0.0004 & 0.013 & 0.0065 & 0.0174 & 0.00 & 0.0016 & 0.0006 & 0.0002 \\
\hline \begin{tabular}{|l|}
10 \\
\end{tabular} & 33.870 & 42 & .0 .002 & 1.34 & 2.32 & 0.0784 & 0.62 & 28.9 & 1.51 & 0.35 & 1.01 & 0.0907 & 0.0118 & 0.0069 & 0.0061 & 0.0018 & 0.0097 & $0.0463 \mid$ & $0.14 \mid$ & $0.0016]$ & 0.0022 & 0.0015 & 0.0016 \\
\hline \begin{tabular}{|l|}
11 \\
\end{tabular} & $34.830 \mid$ & 25 & -0.002 & 1.38 & 2.32 & 0.0715 & 0.64 & 28.42 & 1.47 & 0.311 & 1.221 & 0.0953 & 0.0125 & 0.0072 & $0.0083 \mid$ & 0.0025 & 0.0091 & 0.0535 & $0.14 \mid$ & 0.0017 & 0.0015 & $0.0016 \mid$ & 0.0016 \\
\hline \begin{tabular}{|l|}
122 \\
\end{tabular} & $36.930 \mid$ & 166 & 0.002 & 1.65 & 2.79 & 0.0892 & 0.72 & 24.73 & 1.65 & 0.411 & $1.05 \mid$ & 0.0805 & 0.011 & 0.0059 & 0.0054 & 0.002 & 0.008 & 0.0462 & 0.13 & 0.0017 & $0.0027 \mid$ & 0.0017 & 0.0015 \\
\hline \begin{tabular}{|l|l|}
133 \\
\end{tabular} & $38.330 \mid$ & 9 & 0.0041 & 1.69 & 2.45 & 0.0962 & 0.78 & 27.18 & 1.72 & 0.3 & 0.84 & 0.0827 & 0.0123 & 0.0068 & 0.0048 & .0014 & 0.0072 & 0.0402 & 0.13 & 0.0015 & 0.0016 & $0.0016 \mid$ & 0.0012 \\
\hline \begin{tabular}{|l|}
14 \\
\end{tabular} & 4 & 167 & 0.002 & 1.49 & 3.1 & 0.0691 & 0.66 & 24.14 & 1.57 & 0.48 & 1.72 & 0.12 & 0.0162 & 0.0082 & 0.0111 & 0.0019 & 0.0097 & $0.0525 \mid$ & 0.13 & 0.0017 & $0.0035 \mid$ & $0.0015]$ & 0.0016 \\
\hline \begin{tabular}{|l|}
15 \\
\end{tabular} & 180 & 43 & -0.002 & 1.5 & 2.96 & 0.0798 & 0.71 & 26.68 & 1.73 & 0.43 & 1.79 & 0.13 & 0.0174 & 0.0106 & 0.0124 & .0022 & 0.011 & $\overline{0.0513}$ & $0.14]$ & 0.002 & 0.0023 & 0.0017 & 0.0018 \\
\hline \begin{tabular}{|l|}
16 \\
\end{tabular} & & 168 & 0.002 & 1.44 & & 0.0653 & 0.62 & 24.36 & 1.61 & 0.55 & $1.74 \mid$ & 0.12 & 0.015 & 0.0092 & 0.0108 & 0.0019 & 0.0088 & 0.046 & 0.13 & 0.0018 & 0.0041 & $0.0013 \mid$ & 0.0014 \\
\hline \begin{tabular}{|l|l|} 
\\
\end{tabular} & 330 & 20 & 0.0101 & 1.42 & 2.74 & 0.0596 & 0.63 & 27.53 & 1.66 & 0.5 & & 0.12 & 0.0151 & 0.0 & 0.0136 & 0.0018 & 0.0102 & 0.0508 & 0.14 & 0.00 & & 0.0012 & 0.0011 \\
\hline \begin{tabular}{|l|l|} 
\\
\end{tabular} & & 44 & -0.002 & 1.39 & 3.3. & 0.0853 & 0.65 & 25.2 & 1.84 & 0.33 & 1.76 & 0.13 & 0.0154 & 0.0105 & 0.0074 & 0.0023 & 0115 & 0.0559 & 0.14 & 0.001 & 0.0036 & 013 & 0.0019 \\
\hline \begin{tabular}{|l|}
19 \\
\end{tabular} & 330 & 26 & 0.0032 & 1.49 & 3.2 & 0.1 & 0.73 & 25.71 & 1.79 & 0.28 & 1.2 & 0.095 ] & 0.0133 & $0.0088]$ & 0.0069 & 0.0018 ] & 0.0121 & $0.0418]$ & 0.14 & 0.0016 & 0.0025 & 0.0013 & 0.0019 \\
\hline 20 & & 169 & 0.002 & 1.4 & 3.33) & 0.0941 & 0.63 & 22.43 & 1.64 & 0.39 & 1.51 & 0.14 & 0.0143 & & & & 0.0126 & 0.0445 & 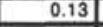 & & & & \\
\hline \begin{tabular}{|l|}
211 \\
\end{tabular} & 50] & 170 & 0.002 & 1.36 & 2.97 & 0.0728 & 0.57 & 21.22 & 1.59 & 0.43 & 1.57 & 0.14 & 0.0157 & 0.0081 & 0.0088 & 0.0018 & 0.0093 & 0.0434 & 0.12 & 0.001 & 0.00 & | 0.001 & 0.0017 \\
\hline \begin{tabular}{|l|}
22 \\
\end{tabular} & 001 & 171 & 0.002 & 1.1 & 2.85 & 0.0613 & 0.49 | & 22.99 & 1.48 & 0.35 & 1.52 & 0.13 & 0.013 & 0.0 & & & 0.0 & 0.0408 & 0.13 & & & | 0.001 & 0.0014 \\
\hline & & 10 & -0.002 & 1.03 & 2.78 & 0.6 & 0.52 & 17.82 & 1.48 & 0.29 & 1.45 & 0.13 & 0.0136 & & & & & 0.08 & & & & & 0.0012 \\
\hline 24 & & 45 & -0.0022 & 1.37 & 2.49 & 0.0856 & 0.58 & 20.85 & 1.45 & 0.24 & 1. & 0.1 & 0.0 & & & & & 0.03 & 0.1 & & & 001 & 0.0014 \\
\hline & & 22 & 0.00222 & 1.7 & 2.53 & 0.0 & 74 & 27.09 & & & & 0.1 & & & & & & & & & & & 0.0016 \\
\hline \begin{tabular}{|l|}
26 \\
\end{tabular} & 52.320 | & 172 & 0.002 & 1.64 & 3.43 & & 0.72 & & 1.53 & 0.36 & & & 0.6 & & & & & & & & & 0. & 0.0014 \\
\hline \begin{tabular}{|l|}
277 \\
\end{tabular} & & 27 & 0.034 & 0.64 & 0.94 & 0.0514 & 1.03 & 31.89 & & & & & & & & & & 0.0 & & & & & -0.0027 \\
\hline \begin{tabular}{|l|}
28 \\
2
\end{tabular} & & 28 & 0.0047 & 0.67 & 0.9 & 0.06 & 0.97 & 30.64 & 1. & 0.2 & 0 & 0.6 & & & & & & & & & & & 0.0 \\
\hline$|29|$ & 54 & 29 & -0.002 & 0.9 & 2.14 & 0.0 & 0.5 & 17.3 & 1.34 & 0.21 & 1 & 0.068 & 0.6 & 0.0 & & & & & & & & & \\
\hline |30 & 54 & 46 & 0.002 & 0.08 & 1,19 & 0.0 & 0.4 & 6.04 & 1.10 & 0.24 & 0.44 & 0.02924 & & & & $=0.00$ & 0.043 & & & & & 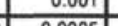 & 0.0007 \\
\hline \begin{tabular}{|l|l|}
31 \\
2
\end{tabular} & 54 & $\frac{173}{2}$ & 0.002 & 0.53 & 0.15 & 0.0314 & 0.26 & $\frac{2.25}{2.55}$ & 0.89 & 0.25 & 0.488 & 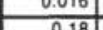 & & & & & & & 0 & & & & \\
\hline (32) & $\frac{50.2000}{57.400}$ & 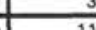 & $=0.00206$ & $\frac{1.38}{1.38}$ & $\frac{4.02}{35}$ & $\frac{10}{10}$ & 0.06 & 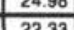 & 1.6 & 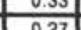 & -4 & 0.16 & 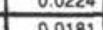 & & & & & & 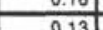 & & & & 0.0019 \\
\hline \begin{tabular}{|l|}
34 \\
34
\end{tabular} & 57.700 & 174 & 0.0002 & 1.53 & 3.49 & & 0.57 & & 174 & 0.38 & & 0.04 & 08 & 0.0 & 0.00 & 0.0 & 0.0 & 0.0 & 0.1 & & 0 & 0.0009 & $\frac{0.0014}{0.0014}$ \\
\hline & 59. & $\overline{47}$ & -0.002 & 1.44 & 4.28 & 0.0732 & 0.64 & 24.37 & 1.91 & 0.52 & 1.95 & \begin{tabular}{l|l|l}
0.19 \\
\end{tabular} & 0.0233 & 0.0 & 0.0 & & 0.0 & 0 & \begin{tabular}{c|c|c|} 
\\
\end{tabular} & & 0.0 & 0.001 & 0.0021 \\
\hline \begin{tabular}{|l|}
36 \\
\end{tabular} & 60.2 & $\overline{48}$ & 0.0045 & 1.93 & 1.93 & 0.11 & 0.84 & 3.86 & 1.34 & 0.26 & 0.0506 & 0.028 & 0.0076 & 0. & 0.0 & & $\overline{0.0}$ & 0.8 & 0.025 & & & & 0.0003 \\
\hline \begin{tabular}{|l|}
37 \\
\end{tabular} & 60.4000 & 16 & 0.0207 & 1.36 & 4.23 & 0. & 0.55 & 95 & 58 & 0.56 & 2.22 & 0.15 & 0. & 0.0 & 0.0 & & 0.0 & & 0.1 & & 0.004 & 0.0000 & $\overline{0.0022}$ \\
\hline \begin{tabular}{|l}
38 \\
\end{tabular} & 61.5 & 175 & 0.6 & 71 & 82 & 0.05 & 0.6 & 16 & 1.87 & 0.82 & 2.16 & 0.14 & 0. & & & & & & 0.1 & & & 0.0009 & \\
\hline 39 & 61.7 & 176 & 0.0102 & 56 & 66 & 0. & 0.58 & 64 & 76 & 0.78 & 2.12 & 0.14 & 0. & 0.0 & & & 0.0 & 0.06 & 0. & & 0.0057 & 001 & 0.00 \\
\hline \begin{tabular}{|l|l} 
\\
\end{tabular} & 63.4000 & 30 & 0.0285 & 42 & 5.55 & 0.8 & 0.59 & 06 & 78 & 0.55 & 2.11 & 0.22 & & & & & & & & & & 009 | & \\
\hline 41 & 64.1 & 177 & 0.023 & 16 & 7.3 & 0.6 & 0.81 & 99 & 2.17 & 1.03 & 2.66 & 0.11 & 0.0389 & 0.02 & 86 & 0.00 & 264 & 0.0765 & 0.12 & 0.0025 & 0.0083 & 0.001 & 0.00 \\
\hline \begin{tabular}{|l|}
42 \\
\end{tabular} & 64.900 & & 0.0482 & 1.21 & 6.33 & 0.0255 & 0.64 & \begin{tabular}{|l|l}
18.93 \\
\end{tabular} & .73 & 0.54 & & & 0.0 & 0.0203 & 0.0169 & 0.0024 & & 0.069 & & & 0.0072 & 0.000 & 0.0028 \\
\hline
\end{tabular}


Table 3 (continued).

\begin{tabular}{|c|c|c|c|c|c|c|c|c|c|c|c|c|c|c|c|c|c|c|c|c|c|c|c|}
\hline & pth (mbs) & mplent & Si & $\overline{A l}$ & $\mathrm{Fe}$ & $\overline{\pi i}$ & $\mathrm{Mg}$ & $\mathrm{Ca}$ & $\mathrm{Na}$ & K & $\mathrm{Mn}$ & $\bar{P}$ & $\mathrm{Cu}$ & $\mathrm{Zn}$ & $\overline{\mathrm{NI}}$ & Co & & $\overline{B a}$ & $\overline{S t}$ & $\overline{Z r}$ & $\bar{B}$ & $\bar{L}$ & La \\
\hline \begin{tabular}{l|l|}
43 & \\
\end{tabular} & 65.390 & 179 & 0.0328 & 1.71 & 5.84 & 0.0336 & 0.72 & 15.85 & 1.99 & 0.9 & 2.72 & 0.14 & 0.0371 & 0.0213 & 0.0167 & 0.0023 & 0.0063 & 0.1 & 0.13 & 0.0025 & 0.0077 & 0.0011 & 0.0031 \\
\hline 44 & 66.960 & 12 & 0.0303 & 1.72 & 6 & 0.0449 & 0.67 & 19.49 & 1.97 & 0.72 & 2.69 & 0.23 & 0.0287 & 0.0213 & 0.0128 & 0.0022 & 0.0122 & 0.0652 & 0.13 & 0.0023 & 0.0072 & 0.0009 & 0.0024 \\
\hline \begin{tabular}{l|l}
45 \\
\end{tabular} & 68.650 & 49 & .0 .002 & 4.83 & 3.12 & 0.38 & 1.1 & 15.05 & 2.42 & 1.61 & 0.14 & 0.14 & 0.0049 & 0.009 & 0.0026 & 0.001 & 0.02 & .0085 & 0.0604 & .0026 & 0.0066 & 0.0019 & 0.001 \\
\hline \begin{tabular}{l|l}
46 \\
\end{tabular} & 69.350 & 21 & .0 .002 & 2.17 & 1.22 & 0.0827 & 0.46 & 5.99 & 0.61 & 0.24 & 0.0381 & 0.0235 & 0.0018 & 0.002 & -0.002 & -0.001 & 0.007 & 0.0033 & 0.0224 & 0.001 & -0.001 & 0.0007 & $\begin{array}{l}0.0005 \\
\end{array}$ \\
\hline 47 & 69.760 & 17 & .0 .002 & 2.14 & 10.79 & 0.12 & 0.9 & 16.14 & 1.86 & 0.87 & 0.55 & 0.31 & 0.0085 & 0.0174 & 0.0082 & 0.0011 & 0.13 & 0.0236 & 0.0826 & 0.0035 & 0.0055 & 0.001 & 0.0027 \\
\hline 48 & 800 & 50 & .0 .002 & 1.93 & 10.13 & 0.11 & 0.81 & 16.71 & 1.7 & 0.75 & 0.76 & 0.32 & 0.0096 & 0201 & 0.0086 & 0.001 & 0.0903 & 0.0217 & 0.0819 & 0.0031 & 0.0058 & 0.001 & 0.0036 \\
\hline 49 & 980 & 19 & 0.0296 & 2.02 & 5.86 & 0.0547 & 0.72 & 17.76 & 1.73 & 0.78 & 2.29 & 0.21 & 0.0455 & .0172 & 0.0119 & 0.0044 & 0.0046 & 0.0475 & 0.1 & 0.0018 & 0.0057 & 0.0009 & 0.0022 \\
\hline 50 & 80 & 180 & 0.002 & 3 & 2.61 & 0.16 & 0.93 & 1.55 & 1.18 & 0.92 & 0.06 & 0.2 & 0.0096 & 0.0058 & 0.002 & 0.0011 & 0.0119 & 0.0067 & 0.0224 & 0.0033 & 0.0013 & 0.0016 & 0.0013 \\
\hline 51 & 2.920 & 31 & 0.0299 & 1.76 & 5.51 & 0.041 & 0.7 & 20.03 & 1.96 & 0.75 & 2.53 & 0.24 & 0.0336 & 0.0211 & 0.013 & 0.0026 & 0.0103 & 0.0623 & 0.13 & 0.0017 & 0.0068 & 0.001 & 0.0022 \\
\hline \begin{tabular}{l|l}
52 \\
\end{tabular} & 60 & 181 & 0.002 & 3.46 & 6.83 & 0.14 & 0.97 & 12.26 & 2.54 & 1.44 & 2.07 & 0.14 & 0.0238 & 0174 & 0.0103 & .0023 & 0.011 & 0.0519 & 0.0912 & 0.0021 & 0.0065 & 0.0011 & 0.0021 \\
\hline 53 & 870 & 33 & 0.0063 & 3.4 & 4.82 & 0.17 & 0.88 & 11.41 & 1.92 & 0.89 & 1.39 & 0.19 & 0.0194 & 0.0127 & 0.006 & 0.0021 & 0.0169 & 0.0325 & 0.0843 & 0.0031 & 0.0016 & 0.0011 & 0.0016 \\
\hline 54 & & 182 & 0.002 & 1.56 & 77 & .0647 & 0.62 & 21.82 & 1.79 & 0.78 & 2.13 & 0.18 & 0.0203 & .0121 & 0.0069 & 0.0019 & 0.0143 & 0.0414 & 0.12 & & .0062 & & .0014 \\
\hline 55 & 000 & 183 & 0.0031 & 2.18 & 4.41 & 0.0826 & 0.64 & 18.32 & 1.92 & 0.97 & 1.96 & 0.16 & 0.0214 & 0.0125 & 0.0072 & 0.002 & 0.0105 & 0.0487 & 0.12 & 0.0024 & 0.0061 & 0.001 & 0.0016 \\
\hline 56 & & 6 & 0.0062 & 2.88 & 5.39 & 0.1 & 0.82 & 15.62 & 2.04 & 0.86 & 2.13 & 0.19 & 0.0228 & 0.0167 & 0.0101 & 0.0025 & 0.0133 & 0.057 & 0.11 & .0024 & 0.0057 & 0.001 & 0.0026 \\
\hline 57 & 460 & 184 & 0.002 & 3.44 & 8 & 0.0741 & 1.08 & 9.57 & 2.76 & 1.64 & 2.99 & 0.18 & 0.0319 & 0.0242 & 0.015 & 0.0051 & 0.0086 & 0.0889 & 0.0911 & 0.0028 & 0.01 & 0.0015 & 0.0031 \\
\hline 58 & 650 & 185 & 0.002 & 2.92 & 9.35 & 0.15 & 1.13 & 14.34 & 2.45 & 1.32 & 38 & 0.24 & 0.0039 & 0.0097 & 0.0059 & 0.0012 & 0.0712 & 0.0259 & 0.0749 & 0.0033 & .0057 & 0.0014 & .0015 \\
\hline 59 & 740 & 23 & .0 .002 & 4.24 & 7.01 & 0.27 & 1.5 & 11.86 & 2.66 & 91 & 0.34 & 0.15 & 0.0095 & 0.0155 & 0.0044 & 0.0012 & 0.0097 & 0.0179 & 0.069 & 0.0029 & 0.0057 & 0.0016 & 0.0016 \\
\hline 60 & & 24 & 0.0062 & 0.75 & 0.98 & .0386 & 0.38 & 3.3 & 1.14 & 0.21 & 0.17 & .0356 & 0.0021 & 0.0026 & 0.0008 & 0.0002 & 0.0035 & 0.0063 & 0.02 & 0.0011 & .0013 & 0.0004 & .0002 \\
\hline 61 & 440 & 51 & 0.0054 & 1.01 & 0.82 & 0.0484 & 0.51 & 2.73 & 1.13 & & & 2277 & 0.0018 & 0.0028 & 0.0009 & 0.0003 & 0.0076 & 0.0047 & 0.016 & 0.0014 & 0.0014 & 0.0006 & 0.0002 \\
\hline 62 & & 32 & 0.0035 & 1.03 & 0.74 & 0.0495 & 0.54 & 2.58 & 1.28 & 0.2 & 0.27 & 0.0233 & 0.0023 & 0.0029 & 0.0009 & 0.0003 & 0.0173 & & 0.0151 & 0.0014 & 0.0014 & 0.0006 & .0002 \\
\hline 63 & 360 & 52 & 0.0037 & 3.03 & 7.79 & 0.12 & 0.94 & 14.09 & 2.48 & 1.39 & 2 & 0.22 & 0.036 & 0.0182 & .0098 & 0.0029 & 0.0108 & & 0.086 & 0.0037 & & & \\
\hline \begin{tabular}{l|l|}
64 \\
\end{tabular} & & 34 & -0.0046 & 2 & 2.48 & 0.1 & 0.81 & 16.29 & 1.72 & $=$ & 0.76 & 0.13 & 0.0133 & 0.0092 & 0.0054 & .0 .0023 & 0.0079 & 0.0266 & 0.0933 & 0.003 & .0 .0023 & 0.0023 & 0.0024 \\
\hline 65 & & 2 & $\begin{array}{l}0.002 \\
\end{array}$ & 1.81 & 3.48 & 0857 & 0.7 & 23.8 & 1.84 & 68 & 2.09 & 0.17 & 0.016 & 0.0101 & 0.007 & 0.0024 & 0.0149 & 0.0331 & 0.13 & 0.002 & 0.0045 & 0.0012 & 0.0014 \\
\hline 66 & & 186 & 0.002 & 1.89 & 3.62 & 0.0864 & 0.62 & 23.19 & 1.86 & 0 & & 0.16 & 0.0126 & 0.0077 & 0.0054 & 0.0025 & 0.0149 & 0.0297 & 12 & 0.0023 & 0.0033 & 0.0013 & 0.0011 \\
\hline 67 & 790 & 13 & 0.0076 & 2.48 & 3.18 & 0.11 & 0.79 & 20.67 & 2.04 & 0.89 & 1.69 & 0.15 & 0.013 & 0.0098 & 0.0071 & 0.0029 & 0.0117 & 0.03 & 0.11 & 0.0024 & 0.0033 & 0.0012 & 0.0014 \\
\hline 68 & & 187 & 0.002 & 3.05 & 3.38 & 0.11 & 0.88 & 18.36 & 2.09 & $\vec{x}$ & 918 & 0.15 & 0.0097 & 0.0069 & 0.0027 & 0.002 & 0.0092 & 0.0247 & 0.0978 & 0.0028 & 0.0024 & 0.0016 & .0012 \\
\hline 69 & & 14 & 0.0037 & 1.6 & 4.08 & 0988 & 0.96 & 3.05 & 1,12 & 0 & 0.16 & .0381 & 0.0093 & 0.0062 & 0.0029 & 0.0013 & 0.007 & 0.0055 & 0.0197 & 0.0009 & 0.0045 & 0.0008 & \\
\hline 70 & & 53 & 0.0054 & 56 & 64 & 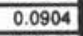 & & 3.68 & 1.32 & 0. & & & & & 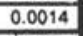 & 05 & $\overline{00}$ & & & & & & 03 \\
\hline 71 & & 1 & 0.0062 & 0.94 & 0.68 & 0.0492 & 0.39 & 2.12 & 1.13 & 0.2 & 0.24 & 0239 | & 0.0074 & 0.0021 & 0.0007 & 0.0004 & 0.0096 & 0.0049 & 0.0123 & 0.0005 & 0.0013 & 0.0003 & 001 \\
\hline 72 & & 40 & 0.0054 & 0 & 96 & 0.0543 & 10 & 2.04 & 0.83 & 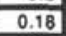 & & 0.0234 & 0.0026 & 0.0023 & 0.0008 & 0.0005 & 0.0036 & 0.004 & 0.011 & 0.0005 & 0.0012 & 0.0004 & .0001 \\
\hline 73 & 000 & 36 & $\begin{array}{l}0.002 \\
\end{array}$ & 2.32 & 3.19 & 0.13 & 0.72 & 12.41 & 2.02 & 0.84 & 1.05 & 0.16 & 0.0124 & 0.0089 & 0.0042 & 0.0023 & 0.0084 & & & & 0.002 & & \\
\hline 74 & & 54 & 0.002 & 1.89 & 2.02 & 0.11 & 0.66 & 5.6 & 1.94 & 0.73 & 0.59 & 0.0873 & 0.0081 & 0.0058 & 0.0032 & 0.0012 & 0.0048 & 0.0137 & 0.0355 & 0.0024 & -0.001 & 0.0011 & 0.0012 \\
\hline 75 & 94.620 & 4 & 0.0058 & 0.76 & 0.79 & 0.0469 & 0.36 & 1.59 & 1.23 & 0.21 & 0.12 & 0.0254 & 0.0038 & 0.002 & 0.0011 & 0.0003 & 0.0018 & & & 0.0007 & 0.0011 & 0.0003 & 0.0002 \\
\hline 76 & 100 & 188 & 0.002 & 0.95 & 0.97 & 0.0499 & 0.38 & 1.85 & 1.17 & 0.4 & 0369 & 0.0262 & 0.0006 & 0.0013 & 0.002 & 0.001 & 0.0019 & 0.0027 & 0.0101 & 0.001 & 0.0012 & 0.0008 & 0.0005 \\
\hline 77 & & 37 & 0.0056 & 0.91 & 0.88 & 0.052 & 0.42 & 1.78 & 1.33 & 0.26 & 0.0 & 0.02 & & & & & 0.002 & & & 0.0009 & & 0.0004 & .0001 \\
\hline 78 & 97.020 & 189 & 0.002 & 2.57 & 4.8 & 0.14 & 0.82 & 17.77 & 2.03 & 1.24 & 1.23 & 0.18 & 0.0138 & 0.0095 & 0.0039 & 0.0023 & 0.012 & 0.0356 & 0.0878 & 0.0029 & 0.0036 & 0.0014 & 0.0011 \\
\hline 79 & 170 & 35 & .0 .002 & 2.46 & 4.72 & 0.16 & 0.89 & 18.83 & 2.24 & 1.2 & 1.3 & 0.2 & & 0.0121 & & & 0.0146 & & & 0.0031 & & 0.0016 & 0.0019 \\
\hline 80 & 103.40 & 38 & .0 .002 & 2.27 & 4.81 & 0.15 & 0.91 & \begin{tabular}{|l|}
20.46 \\
\end{tabular} & 2.08 & 0.97 & 1.27 & 0.25 & 0.0159 & 0.011 & 0.0048 & 0.0021 & 0.0194 & 0.0391 & 0.0963 & 0.0024 & 0.0031 & 0.0016 & 0.0014 \\
\hline 81 & 103.65 & 190 & 0.002 & 2.97 & 6.37 & 0.23 & 1.13 & 12.09 & 2.25 & 0.84 & 1.37 & 0.17 & 0.0204 & 0.0117 & 0.0059 & 0.0025 & 0.016 & 0.0283 & 0.068 & 0.0034 & 0.0033 & 0.0018 & 0.0012 \\
\hline 82 & 104.52 & 55 & 0.0052 & 2.41 & 5.02 & 0.13 & 0.92 & \begin{tabular}{|l|}
20.96 \\
\end{tabular} & 2.18 & 1.11 & 1.17 & 0.27 & 0.0212 & 0.0131 & 0.0053 & 0.0014 & 0.0179 & 0.0466 & 0.0951 & 0.0034 & 0.0038 & 0.0017 & 0.0022 \\
\hline 83 & 104.80 & 39 & 0.003 & 2.42 & 5.97 & 0.14 & 0.98 & \begin{tabular}{|l|}
17.43 \\
\end{tabular} & 2.15 & 1.19 & 1.55 & 0.23 & 0.0277 & 0.0167 & 0.0079 & 0.0021 & 0.0213 & 0.0556 & 0.091 & 0.003 & 0.0058 & 0.0026 & 0.002 \\
\hline
\end{tabular}




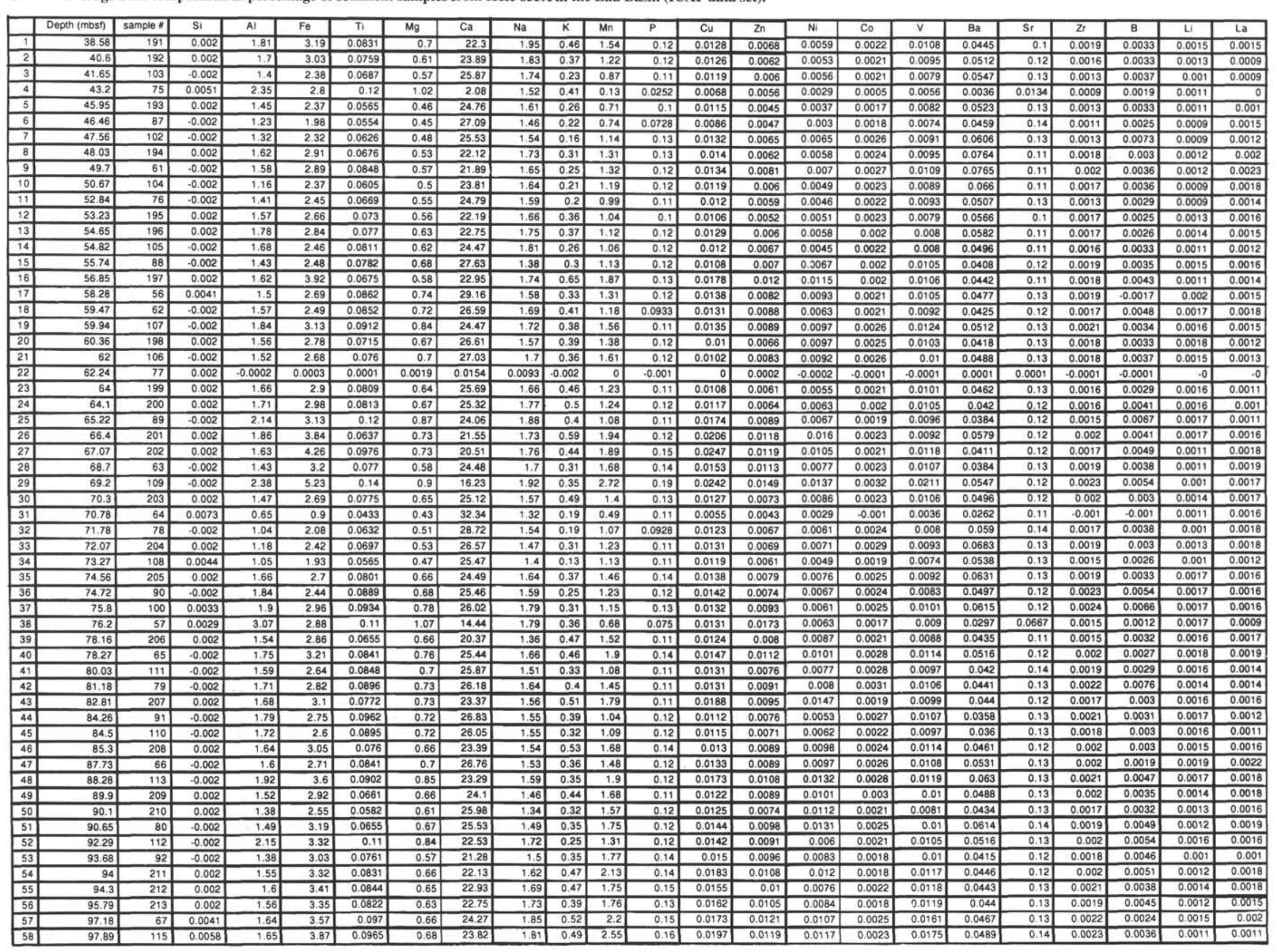




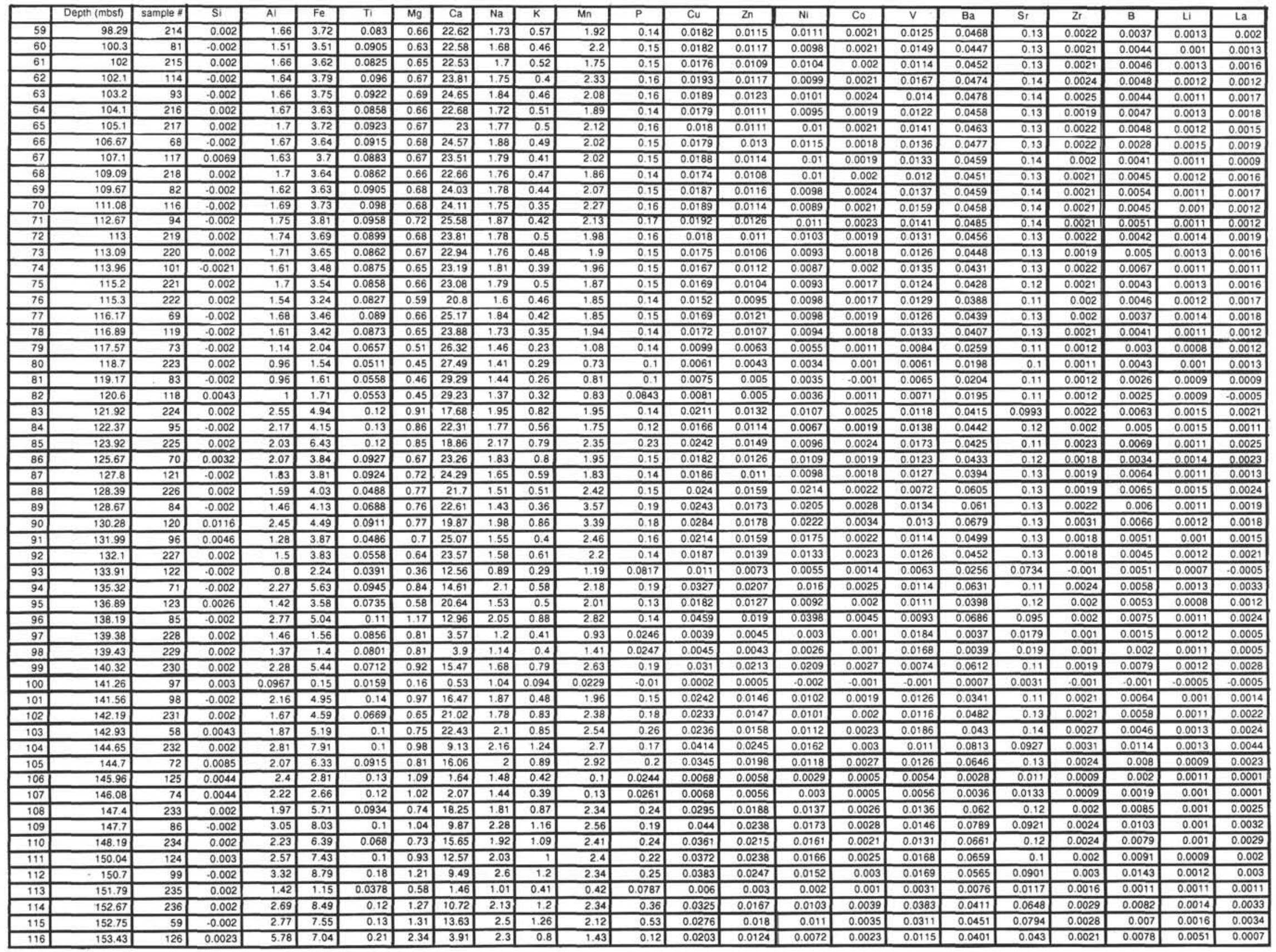


Table 5. Average bulk composition in percentage of sediment samples from Hole 838A in the Lau Basin (ICAP data set).

\begin{tabular}{|c|c|c|c|c|c|c|c|c|c|c|c|c|c|c|c|c|c|c|c|c|c|c|c|}
\hline & Depth (mbsf) & ample of & $\overline{\mathrm{Si}}$ & $\overline{A l}$ & $\mathrm{Fe}_{\theta}$ & $\overline{T i}$ & $M_{g}$ & $\mathrm{Ca}$ & $\mathrm{Na}$ & $\bar{K}$ & $M n$ & $\bar{p}$ & $\mathrm{Cu}$ & $2 n$ & $\overline{\mathrm{Ni}}$ & co & v & $8 a$ & $\overline{\mathrm{St}}$ & $\overline{2 r}$ & $\bar{B}$ & $\overline{\mathrm{Li}}$ & La \\
\hline 7 & 34.7 & 128 & 0.009876 & 0.21 & 0.21 & 0.02041 & 0.13 & 0.36 & 0.72 & 0.04188 & 0.01221 & 0.01023 & 0.00057 & 0.0005 & .0 .0002 & .0 .0001 & 0.00065 & 0.00106 & 0.00193 & .0 .0001 & 0.00036 & 0.00008 & 0.00006 \\
\hline 2 & 37.5 & 127 & \begin{tabular}{|l|}
0.009959 \\
\end{tabular} & 0.21 & 0.23 & 0.02138 & 0.15 & 0.51 & 0.87 & 0.04898 & 0.00866 & 0.00895 & 0.0006 & 0.00055 & .0 .0002 & 0.0001 & 0.00067 & 0.00077 & 0.00244 & 0.00012 & 0.00049 & 0.00008 & -0.00005 \\
\hline 3 & 42.07 & 130 & -0.002 & 2.36 & 3.02 & 0.11 & 0.91 & 21.52 & 1.75 & 0.28 & 1.05 & 0.08905 & 0.01334 & .00758 & 0.00467 & 0.00231 & 0.00948 & 0.02921 & 0.1 & 0.0014 & 0.00315 & 0.00151 & 0.00073 \\
\hline 4 & 45.4 & 142 & 0.01089 & 0.33 & 0.27 & 0.02012 & 0.22 & 0.31 & 1.25 & 0.06807 & 0.06434 & 0.02206 & 0.00072 & 0.00065 & 0.00034 & 0.00014 & 0.00085 & 0.00231 & 0.0019 & 0.00012 & 0.00051 & 0.00007 & 0.00009 \\
\hline 5 & 49 & 129 & 0.009846 & 0.38 & 0.35 & 0.02456 & 0.33 & 0.38 & 1.59 & 0.08147 & 0.12 & 0.02158 & 0.001 & 000 & 0.00048 & 0.00019 & 0.0012 & 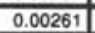 & 0.0023 & 0.00014 & 0.0008 & 0.00007 & 0005 \\
\hline 6 & & 132 & 0.01093 & 0.27 & 0.42 & 0.03394 & 0.19 & 0.42 & 1.16 & 0.05868 & 0.03956 & 0.04394 & 0.00072 & 0.00101 & -0.0002 & 0.00013 & 0.00127 & 0.0022 & 0.00208 & -0.0001 & 0.00054 & -0.00005 & 0.00011 \\
\hline 7 & & 131 & \begin{tabular}{|l|}
0.009328 \\
\end{tabular} & 0.43 & .53 & \begin{tabular}{l|}
0.04028 \\
\end{tabular} & 0.3 & 0.53 & 1.42 & 0.07474 & 0.1 & 0.04971 & 0.00114 & 0.00149 & 0.00031 & 0.00025 & 0.00179 & 0.00193 & 0.00235 & 0.00014 & 0.00074 & 0.00009 & 0.00009 \\
\hline .8 & 60 & 11 & \begin{tabular}{|l|}
0.013523 \\
\end{tabular} & 0.42 & 0.33 & 0.02184 & 0.28 & 0.37 & 1.63 & 0.08607 & 0.08234 & 0.0264 & 0.00088 & 0.00632 & -0.00026 & 0.00014 & 0.001 & 0.00198 & 0.00221 & $\frac{0.00013}{-0.03}$ & 0.00084 & 0.00011 & 0.00009 \\
\hline 9 & 63.1 & 134 & 0.007949 & 0.78 & 0.59 & 0.03443 & 0.34 & 0.6 & 1.57 & 0.09994 & 0.28 & 0.04139 & 0.00192 & 0.00152 & 0.00079 & 0.00046 & 0.00188 & 0.00456 & 0.00346 & 0.00017 & 0.00094 & 0.00012 & 0.00009 \\
\hline 10 & & & \begin{tabular}{l|l}
-0.002 \\
\end{tabular} & 2.3 & 2.82 & 0.1 & 0.83 & 21.99 & 1.62 & 0.31 & & 0.07533 & 0.01092 & 0.00786 & 0.00296 & 0.00235 & 0.00766 & 0.03216 & 0.1 & 0.00124 & 0.00381 & 0.0013 & 0.00081 \\
\hline 11 & 66.89 & 133 & -0.002 & 2.5 & 2.98 & 0.11 & 1.05 & 18.13 & 1.64 & 0.29 & 0.84 & 0.071 & 0.01068 & 0.00838 & 0.00302 & 0.00219 & 0.00846 & 0.02941 & 0.08201 & 0.00123 & 0.00418 & 0.0014 & 0.00067 \\
\hline 12 & & 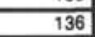 & sit & 4.18 & 10 & 0.0263 & 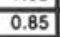 & 2.52 & 0.61 & 0.04558 & & 0.00726 & & 0.00137 & 0.00147 & 0.00077 & 0.00249 & 0.0014 & 0.0063 & 0.00013 & 0.00032 & 0.00013 & .0 .00005 \\
\hline \begin{tabular}{l|l|}
13 \\
\end{tabular} & 76.75 & 135 & 0.002107 & 5.38 & 6.87 & 0.18 & 1.73 & 1.5 & 3.19 & 0.67 & 1.97 & 0.11 & 0.01919 & 0.01464 & 0.00898 & 0.00711 & 0.01025 & 0.03626 & 0.02525 & 0.00197 & 0.00742 & 0.00195 & 0.0009 \\
\hline 14 & & 7 & 0.006362 & 1.89 & 2.21 & 0.12 & 0.86 & 0.66 & 1.62 & 0.59 & 8 & 0.01972 & 0.00 & 0.00516 & 0.00039 & 0.00 & 0.00 & & 0.00 & 0.00068 & 0.00 & 0.0 & 0.0 \\
\hline 15 & 86.19 & 138 & 0.00759 & 1.04 & 0.68 & 0.05516 & 0.42 & 0.47 & 1.09 & 0.12 & 0.00922 & 0.00965 & 0.00126 & 0.00156 & -0.0002 & 0.00013 & 0.00156 & 0.00389 & 0.00238 & 0.0003 & 0.00086 & 0.00044 & 0.00005 \\
\hline 16 & & 1 & 0.0103 & 0.56 & 0.41 & 0.0353 & 0.25 & 0.42 & 1.22 & 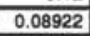 & 000704 & 0.01085 & 0.00086 & 0.00098 & 0.0002 & 0.00 & 0.00098 & 0.00 & 0.00237 & 0.00017 & 0.00089 & 0.00022 & 000 \\
\hline  & 95.92 & 140 & 0.008686 & 1.3 & 0.74 & 0.06227 & 0.34 & 0.8 & 0.83 & 0.09354 & 0.01356 & 0.01676 & 0.00153 & 0.00158 & -0.0002 & 0.00021 & 0.0019 & 0.00383 & 0.00304 & 0.00026 & 0.00074 & 0.00035 & 0.00006 \\
\hline
\end{tabular}

Notes: Depths are in meters below seafloor. Negative values indicate concentrations below detection limit. 
Table 6. Average bulk composition in percentage of sediment samples from Hole 839A in the Lau Basin (ICAP data set).

\begin{tabular}{|c|c|c|c|c|c|c|c|c|c|c|c|c|c|c|c|c|c|c|c|c|c|c|c|}
\hline & $\operatorname{th}$ (mbsi) & mple " & Si & Al & $\mathrm{Fe}$ & $\mathrm{Ti}$ & $\mathrm{Mg}$ & $\mathrm{Ca}$ & $\mathrm{Na}$ & $\bar{K}$ & $\mathrm{Mn}$ & $P$ & $\mathrm{Cu}$ & $2 n$ & $\mathrm{Ni}$ & $\mathrm{Co}_{0}$ & $\bar{v}$ & Ba & St & Zr & $\bar{B}$ & Li & ta \\
\hline 1 & $\begin{array}{ll}42.9 \\
\end{array}$ & 257 & 0.002 & 1.91 & 3.17 & 0.12000 & 0.82 & 21.89 & 1.74 & 0.49 & 0.8 & 0.13 & 0.01141 & 0.00583 & 0.00372 & 0.00105 & 0.01154 & 0.0428 & 0.12 & 0.00137 & 0.00489 & 0.00165 & 0.00183 \\
\hline 4 & \begin{tabular}{ll|}
43.2 \\
\end{tabular} & 258 & 0.002 & 2.28 & 3.89 & 0.13000 & 0.96 & 16.82 & 1.82 & 0.51 & 0.81 & 0.12 & 0.01271 & 0.00659 & 0.00393 & 0.00173 & 0.01386 & \begin{tabular}{l|l|}
0.0435 \\
\end{tabular} & 0.08955 & 0.00129 & 0.00467 & 0.00149 & 0.00186 \\
\hline 3 & 43.79 & 259 & 0.002 & 1.82 & 2.81 & 0.100000 & 0.78 & 10.13 & 1.44 & 0.41 & 0.86 & 0.09876 & 0.00889 & 0.00527 & 0.00533 & 0.00255 & 0.00903 & 0.03328 & 0.05536 & 0.001 & 0.00425 & 0.00117 & 0.00084 \\
\hline 4 & 4.41 & 30 & 0.002 & 2.03 & 3.39 & 0.100000 & 0.83 & 19.01 & 1.79 & 0.48 & 0.99 & 0.12 & 0.01287 & 0.00721 & 0.00583 & 0.00208 & 0.01091 & 0.04181 & 0.1 & 0.00111 & 0.00757 & 0.0016 & 0.00182 \\
\hline 5 & 49.06 & \begin{tabular}{l|l}
153 \\
\end{tabular} & 0.00967 & 0.96 & 0.49 & 0.026270 & 0.31 & 1.47 & 0.88 & 0.09195 & 0.09648 & 0.01965 & 0.01007 & 0.0016 & 0.00042 & 0.00029 & 0.00347 & 0.00326 & .00648 & 0.00022 & 0.00058 & 0.00024 & 0.00009 \\
\hline 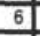 & \begin{tabular}{|l|l|}
51.63 \\
\end{tabular} & 44 & 0.00718 & 0.94 & 0.48 & 0.026190 & 0.32 & 1.49 & 1.05 & 0.08956 & 0.09649 & 0.0207 & 0.00939 & 0.00147 & 0.00041 & 0.00027 & 0.00351 & 0.00345 & 0.00668 & 0.00021 & 0.00071 & 0.00022 & 0.00012 \\
\hline 7 & \begin{tabular}{l|l}
52.2 \\
\end{tabular} & 261 & \begin{tabular}{l|l}
0.002 \\
\end{tabular} & 0.54 & 0.38 & 0.018240 & 0.28 & 1.25 & 0.97 & 0.22 & 0.08094 & 0.01 & 0.00034 & 0.00028 & \begin{tabular}{l|l|}
0.002 \\
\end{tabular} & 0.001 & 0.00127 & 0.00318 & 0.00598 & 0.001 & 0.00297 & 0.00059 & 0.0005 \\
\hline 8 & $\begin{array}{l}54.6 \\
\end{array}$ & 6 &  & 2.14 & 0.74 & 0.038110 & 0.38 & 2.18 & 1.02 & 0.09912 & 0.07159 & 0.0236 & 0.00403 & 0.00178 & 0.00071 & 0.00043 & 0.00286 & 0.00392 & 0.00989 & 0.00028 & 0.0006 & 0.00028 & 0001 \\
\hline 9 & 56.61 & 262 & 0.002 & 0.84 & 0.74 & 0.031200 & 0.46 & 1.97 & 0.96 & 0.29 & 0.15 & 0.01869 & 0.00209 & 0.0018 & \begin{tabular}{l|l}
0.002 \\
\end{tabular} & 0.001 & 0.00246 & 0.00507 & 0.00999 & 0.001 & 0.00145 & 0.00069 & 0.0005 \\
\hline 10 & 79 & 55 & 0.00567 & $\begin{array}{l}1.57 \\
\end{array}$ & 1.39 & 0.051830 & 0.76 & 3.29 & 1.16 & 0.24 & 0.22 & 0.03101 & 0.00554 & 0.00375 & 0.00148 & 0.00065 & 0.00476 & 0.00728 & 0.0173 & 0.00075 & 0.00179 & 0.00068 & 0.00025 \\
\hline 111 & 61.18 & 53 & 0.002 & 0.74 & 0.62 & 0.026270 & 0.42 & 1.58 & 1.12 & 0.27 & 0.09869 & 0.01116 & 0.00131 & 0.00091 & 0.002 & 0.001 & 0.00192 & 0.00411 & 0.00808 & 0.001 & 0.00192 & 0.00069 & 0.0005 \\
\hline 12 & 2.1 & ;4 & 0.002 & 0.79 & 0.68 & 0.028000 & 0.44 & 1.71 & 1.13 & 0.28 & 0.1 & 0.01048 & 0.00152 & 0.00093 & 0.002 & 0.001 & 0.00206 & 0.00417 & 0.00866 & 0.001 & 0.00115 & 0.00064 & 0.0005 \\
\hline 13 & \begin{tabular}{l|}
62.3 \\
\end{tabular} & 265 & 0.002 & 0.85 & 0.75 & 0.030640 & 0.48 & 1.86 & 1.18 & 0.26 & 0.11 & 0.01725 & 0.00188 & 0.00086 & 0.002 & 0.001 & 0.00239 & 0.0044 & 0.0095 & 0.001 & 0.001 & 0.00069 & 0.0005 \\
\hline 14 & \begin{tabular}{l|l}
64.4 \\
6
\end{tabular} & 158 & 0.00805 & 0.97 & 0.42 & \begin{tabular}{|l|}
0.025150 \\
\end{tabular} & 0.29 & 1.25 & 1.11 & 0.07325 & 0.04858 & 0.01902 & 0.00219 & 0.00133 & 0.00036 & 0.00026 & 0.00183 & 0.00341 & 0.00545 & 0.00016 & 0.00058 & 0.00016 & 0.00005 \\
\hline 15 & 65.25 & 146 & 0.0088 & 0.84 & 0.59 & 0.025640 & 0.37 & 1.5 & 0.92 & 0.11 & 0.08285 & 0.01633 & 0.00235 & 0.00166 & 0.00032 & 0.00028 & 0.00208 & 0.00374 & 0.00752 & 0.0003 & 0.0007 & 0.00027 & 0.0001 \\
\hline 16 & \begin{tabular}{l|l|}
66.1 \\
\end{tabular} & 266 & $\begin{array}{ll}0.002 \\
\end{array}$ & 0.59 & 0.45 & 0.020350 & 0.33 & 1.19 & 1.12 & 0.24 & 0.06104 & 0.01238 & 0.00057 & 0.0002 & 0.002 & 0.001 & 0.00137 & 0.00309 & 0.00601 & 0.001 & 0.001 & 0.00059 & 0.0005 \\
\hline 17 & 68.2 & 157 & .00773 & 0.76 & 0.52 & 0.022870 & 0.36 & 1.36 & 1.2 & 0.1 & 0.06591 & 0.0153 & 0.00199 & 0.00138 & 0.00029 & 0.00023 & 0.00172 & 0.00345 & 0.00706 & 0.00025 & 0.00076 & 0.00024 & 0.00007 \\
\hline 18 & $\begin{array}{ll}70.3 \\
\end{array}$ & 77 & 0.002 & 0.72 & 0.36 & 0.020830 & 0.22 & 1.24 & 0.88 & 0.18 & 0.0335 & 0.01911 & 0.00023 & 0.0012 & $\begin{array}{ll}0.002 \\
\end{array}$ & 0.001 & 0.00125 & 0.0028 & 0.00564 & 0.001 & 0.001 & 0.00053 & 0.0005 \\
\hline 19 & 70.65 & 151 & 0.01015 & 0.87 & 0.35 & \begin{tabular}{|c|}
0.021890 \\
\end{tabular} & 0.26 & 1.24 & 1.38 & 66633 & 0.02954 & 0.02125 & 0.00157 & 0.00135 & 0.00037 & 0.00025 & 0.00143 & 0.00269 & 0.00582 & 0.0001 & 0.00054 & 0.00000 & 0.00008 \\
\hline 20 & 71.59 & 8 & 0.002 & 0.73 & 0.42 & 0.025950 & 0.26 & 1.35 & 1.17 & 0.2 & 0.03241 & 0.0221 & 0.00042 & 0.00101 & 0.002 & 0.001 & 0.00161 & 0.00369 & 0.00605 & 0.001 & 0.00118 & 0.0005 & 0.0005 \\
\hline 21 & 73.1 & 69 & 0.002 & 0.84 & 0.43 & \begin{tabular}{|l|}
0.026270 \\
\end{tabular} & 0.26 & 1.47 & 1.16 & 0.19 & 0.03651 & 0.02274 & 0.00036 & 0.0002 & 0.002 & 0.001 & 0.00153 & 0.00317 & 0.00647 & 0.001 & 0.001 & 0.0005 & 0.0005 \\
\hline 22 & 74.5 & 147 & 0.01031 & 1.19 & 0.49 & 0.031030 & 0.26 & 1.6 & 0.95 & 0.05287 & 0.03362 & 0.02957 & 0.00199 & 0.00187 & 0.00032 & 0.00027 & 0.00201 & 0.00373 & 0.00667 & 0.00012 & 0.00043 & 0.00013 & 0.00005 \\
\hline 23 & 81.29 & 270 & 0.002 & 3.66 & 1.02 & 0.064140 & 0.36 & 3.27 & 1.15 & 0.21 & 0.02704 & 0.03828 & 0.00173 & 0.04655 & 0.002 & 0.001 & 0.00405 & 0.00464 & 0.01208 & 0.001 & 0.001 & 0.00064 & 0.0005 \\
\hline 24 & 37 & 160 & 0.01096 & \begin{tabular}{|l|}
3.73 \\
\end{tabular} & 0.78 & \begin{tabular}{|l|}
0.046190 \\
\end{tabular} & 0.3 & 2.3 & 1.33 & 0.08582 & 0.00953 & 0.02484 & 0.00161 & 0.00175 & 0.00033 & 0.00021 & 0.0032 & 0.00183 & 0.00904 & 0.00012 & 0.00073 & 0.0002 & 0.00005 \\
\hline 25 & 84.66 & 149 & 0.00913 & 4.55 & 0.65 & \begin{tabular}{|l|l}
0.032170 \\
\end{tabular} & 0.29 & 2.54 & 0.47 & 0.03262 & 0.02021 & 0.02341 & 0.00309 & 0.00133 & 0.00036 & 0.00027 & 0.00262 & 0.0 & .00938 & .00012 & .00038 & 0.00016 & 0.00005 \\
\hline 26 & 4 & 71 & $\begin{array}{ll}0.002 \\
\end{array}$ & 5.05 & 1.16 & \begin{tabular}{|l|l|}
0.068890 \\
\end{tabular} & 0.48 & 3.74 & 1.17 & 0.2 & 0.02154 & 0.0369 & 0.0012 & 0.00097 & 0.002 & 0.001 & 0.00552 & 0.00308 & 0.01376 & 0.001 & 0.001 & 0.00053 & 0.0005 \\
\hline 27 & \begin{tabular}{l|l|}
86.7 \\
\end{tabular} & 272 & \begin{tabular}{l|l}
0.002 \\
\end{tabular} & 2.32 & 3.75 & 0.12000 & 0.84 & 19.31 & 1.53 & 0.47 & 1.25 & 0.19 & 0.02638 & 0.00901 & 0.00803 & 0.004 & 0.01001 & 0.05764 & 0.1 & 0.00123 & 0.00591 & 0.0016 & 0.00189 \\
\hline 28 & 86.9 & & 0.002 & 2.4 & $\begin{array}{l}5.31 \\
\end{array}$ & 0.12000 & 0.85 & 16.63 & 1.4 & 0.42 & 5 & 0.12 & 0.01263 & 0.00704 & 0.00448 & 0.0013 & 0.00918 & 0.03848 & 0.08579 & 0.00117 & 0.00529 & 0.00155 & 0.0014 \\
\hline 29 & 87.72 & 159 & 0.00408 & 2.98 & 2.1 & \begin{tabular}{|c|}
0.090380 \\
\end{tabular} & 1.08 & 4.1 & 1.28 & 0.21 & 0.93 & 0.03094 & 0.01283 & 0.00774 & 0.00286 & 0.0017 & 0.02297 & 0.0141 & 0.02233 & 0.00084 & 0.00166 & 0.00072 & 0.00053 \\
\hline 30 & 88.4 & 7 & 0.002 & 2.57 & 3.86 & 0.13000 & 1.05 & 17.36 & 1.6 & 0.73 & 0.5 & 0.14 & 0.00646 & 0.00639 & 0.00454 & 0.00164 & 0.01335 & 0.0345 & 0.0845 & 0.001 & 0.00699 & 0.00197 & 0.00116 \\
\hline 31 & 89.66 & 148 & 0.0039 & 2.99 & 4.35 & 0.16000 & 1.12 & 18.91 & 1.93 & 0.58 & 0.57 & 0.12 & 0.00805 & 0.00811 & 0.00419 & 0.00242 & 0.02618 & 0.04609 & 0.09225 & 0.00212 & 0.00787 & 0.00232 & 0.00103 \\
\hline 32 & 90.94 & 151 & 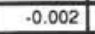 & 3.06 & 4.5 & 0.15000 & 1.15 & 18.32 & 1.91 & 0.58 & 0.55 & 0.11 & 0.00825 & 0.00826 & 0.00485 & 0.00238 & 0.02498 & 0.04684 & 0.09174 & 0.00206 & 0.00789 & 0.002 & 0.00112 \\
\hline 33 & \begin{tabular}{|c|}
92.5 \\
\end{tabular} & 275 & 0.002 & 2.38 & 3.7 & 0.11000 & 0.94 & 17.32 & 1.67 & & 1.46 & 0.14 & 0.0175 & 0.00781 & 0.01029 & 0.00383 & 0.01134 & 0.05076 & 0.09534 & 0.00129 & 0.00559 & 0.00176 & 0.00179 \\
\hline 34 & $\begin{array}{l}92.9 \\
\end{array}$ & 276 & 0.002 & 3.67 & 3.99 & 0.12000 & 1.7 & 17.25 & 1.8 & 0.48 & 0.85 & 0.11 & 0.0145 & 0.00767 & 0.00532 & 0.00222 & 0.01436 & 0.03854 & 0.0833 & 0.001 & 0.00539 & 0.0016 & 0.00148 \\
\hline 35 & & 15 & .0 .002 & 3.58 & 4.54 & 0.13000 & 1.35 & 14. & 7 & & & 0.11 & & 0.00 & 0.00608 & 0.00 & 0.01 & & 0.08013 & 0.00159 & 0.00558 & 0.00167 & 0.00112 \\
\hline 36 & 94.75 & 277 & 0.002 & 1.32 & 1.63 & \begin{tabular}{|l|}
0.084860 \\
\end{tabular} & 0.64 & 4.55 & 1.11 & 0.44 & 0.14 & 0.02157 & 0.00418 & 0.00296 & 0.002 & 0.001 & 0.00831 & 0.00969 & 0.02295 & 0.001 & 0.00261 & 0.00117 & 0.00073 \\
\hline 37 & 96.68 & 278 & 0.002 & 0.45 & 0.44 & \begin{tabular}{|l|}
0.026670 \\
\end{tabular} & 0.24 & 0.89 & 0.82 & 0.26 & 0.02558 & 0.01 & 0.0002 & 0.0002 & 0.002 & 0.001 & 0.00166 & 0.00216 & 0.00446 & 0.001 & 0.001 & 0.00053 & 0.0005 \\
\hline 38 & 98.38 & 150 & 0.01076 & 0.42 & 0.25 & 0.018550 & 0.17 & 1.49 & 0.72 & 0.05116 & 0.03465 & 0.0092 & 0.00081 & 0.00086 & 0.00045 & 0.00011 & 0.00119 & 0.00188 & 0.00577 & 0.0001 & 0.00055 & 0.00013 & .0 .00005 \\
\hline 39 & $\begin{array}{l}99.55 \\
\end{array}$ & 161 & 00429 & 3.13 & 1.67 & 0.085550 & 0.75 & 3.16 & 0.98 & 0.13 & 0.13 & 0.01799 & 0.00613 & 0.00305 & 0.00114 & 0.00098 & 0.01296 & 0.00681 & 0.01132 & 0.00055 & 0.00093 & 0.00046 & 0.00021 \\
\hline
\end{tabular}

Notes: Depths are in meters below seafloor. Negative values indicate concentrations below detection limit. 
A.

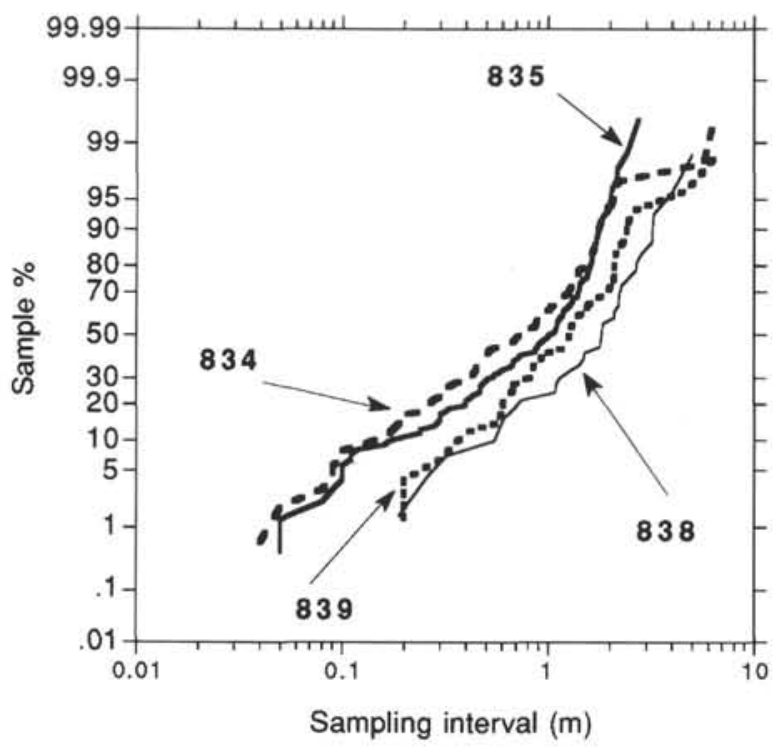

B.

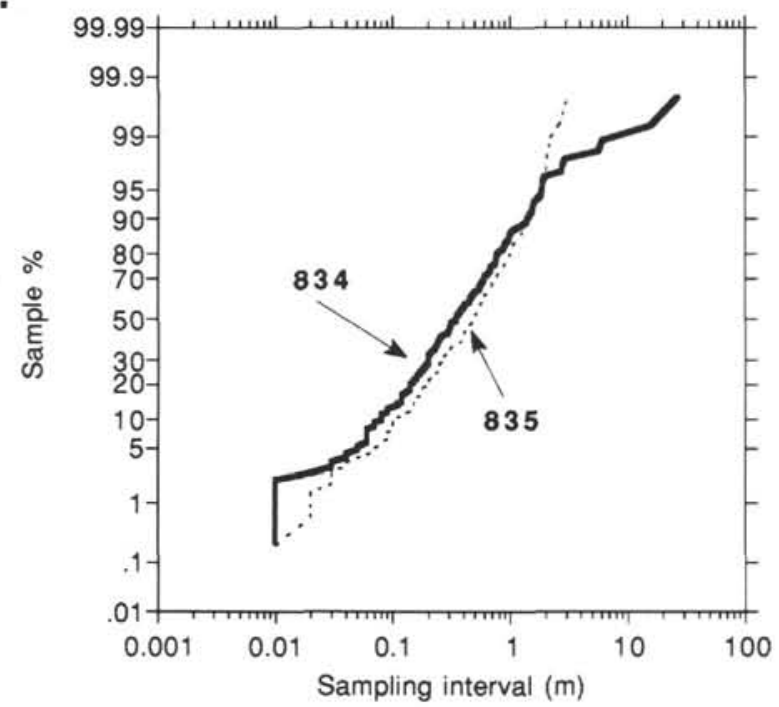

Figure 2. Cumulative distributions of ICAP and HK sampling intervals for Leg 135 sites. A. ICAP analyses. B. HK data set.

$\mathrm{HCl}$ (Thompson and Walsh, 1989). Accuracy was checked with the use of certified international reference materials, along with in-house reference materials. No systematic errors were observed, and accuracy was better than $5 \%$ for elements studied. Analytical precision was checked with the use of duplicate samples and was found to be better than $3 \%$ relative for the elements studied. The analytical results and a detailed description of the analytical method are given in Hodkinson and Cronan (this volume).

The results of both sets of analyses are presented in Figure 3. It should be noted that the three-acid attack used on the HK data set will dissolve all the sediment, including detrital volcanic material and volcanic glass, feldspar, and so forth. The $\mathrm{HNO}_{3}$ attack will not dissolve these resistant detrital phases and thus any potassium associated with such phases will not be taken into solution. This may lead to bias between the two data sets, particularly where K-rich volcaniclastic material predominates. This is borne out by a comparison of the statistics of the two data sets. The HK data set values are on average $0.2 \%$ higher than the ICAP data, with more extreme high values (the maximum value is twice that of the ICAP data set).

\section{PETROPHYSICAL DATA}

The background to the wireline-logging runs at Sites 834 and 835 are given in Parson, Hawkins, Allan, et al. (1992).

In Hole 834B the total gamma response (SGR) from the geochemical logging suite was recorded in open hole throughout the section of interest and these data have been used in this investigation. Pipe base was at 44 mbsf during this run and logging speed was $183 \mathrm{~m} / \mathrm{hr}$ $(600 \mathrm{ft} / \mathrm{hr})$.

In Hole $835 \mathrm{~B}$ the pipe base was at $50 \mathrm{mbsf}$ and the SGR from the "seismo-strat" tool was recorded openhole over the section of interest. Logging speed was greater in this hole $(274 \mathrm{~m} / \mathrm{hr}[900 \mathrm{ft} / \mathrm{hr}])$ which reduces the resolution of the tool somewhat. However, because of the increased sedimentation rate at this site, the logging speed effect is not considered critical.

Environmental corrections were conducted for hole size and borehole fluid (seawater). The corrections affect peak amplitudes rather than peak locations and thus have minimal effect on the Walsh analyses. Fourier analysis, which is influenced by amplitude changes, will be affected by correction in a rugose hole. The Walsh analyses are however affected by the fact that after detrending there is often low amplitude noise close to zero. This noise will produce high frequency artifacts on recoding to +1 and -1 as can be seen in Figure 7 . One possible way around this would be to include a low-pass filter before recoding. The poor match between Walsh spectra from Holes $834 \mathrm{~B}$ and $835 \mathrm{~B}$ may be a result of this phenomena.

\section{CYCLICITY ANALYSIS}

The spectral analysis of time-series data from geological sections and boreholes has been discussed by many people. Good discussions are to be found in Schwarzacher $(1975,1987)$, Smith (1989), and Weedon (1989, 1991). Weedon (1991) discusses both sequency (Walsh) and frequency (Fourier) analyses. A recent discussion of cyclicity in deep marine systems is to be found in Golovchenko et al. (1992).

The programs used for the analysis were adapted from Graham Weedon's Fortran programs, Weedon (1991). These programs are well documented and easily adapted for different circumstances. The actual Walsh and Fourier generation routines were adapted from programs published by Kanasewich (1981) and Davis (1973).

The procedure for sequency and frequency analyses will be discussed below.

All three data series (SGR, ICAP, and HK) were converted from depth to geological time, assuming both (1) a linear depth-time relationship between magnetic reversal depths in the two holes, and (2) a nonlinear depth-time relationship based on $\mathrm{K}: \mathrm{Ca}$ ratios. The common procedure of conducting the analysis in depth and then converting the spectra to time implicitly assumes equal sedimentation rates throughout the section (for pre-Pliocene data); on the other hand, by converting explicitly before the spectral analysis, a change in average sedimentation rate at $3.01 \mathrm{Ma}$ (Kaena reversal) can be incorporated without any difficulty. Converting before analysis also enables resampling to be conducted in time, giving equal-time sampled sections.

An apparent inverse relationship between calcium and potassium is present at both sites (Fig. 4). This suggests that one may be able to adjust the sedimentation rate as a function of potassium/calcium ratio by assuming that a low background sedimentation rate as recorded by the calcium nannofossil ooze is increased as the potassium content increases (because of volcanic sand input to the system). This possibility was examined by normalizing the potassium and calcium values to a proportion of the total in each well. The resulting value " $\mathrm{K} /(\mathrm{K}+$ $\mathrm{Ca}$ " varies from zero to unity and may be taken as indicating relative sedimentation rate. Figure 5 shows how this relative sedimentation rate relates to the calcium content for both wells and how the sedimentation rates compare between wells. To convert the estimated relative sedimentation rate to an absolute value requires knowledge 
of the total time and depth windows obtained from paleomagnetic reversal ages and depths. The conversion involves the solution of $N$ simultaneous equations where $N$ is the number of $\mathrm{K}$ and $\mathrm{Ca}$ values measured from ICAP analysis over the interval in question. The equation set is as follows:

$$
\begin{gathered}
1 . t_{1}-C_{1} t_{2}+0 . t_{3}+\ldots+0 . t_{n-1}+0 . t_{n}=0 \\
0 . t_{1}-1 t_{2}-C_{2} \cdot t_{3}+\ldots+0 . t_{n-1}+0 . t_{n}=0 \\
\bullet \\
\bullet \\
\bullet . t_{1}-0 . t_{2}+0 . t_{3}+\ldots+1 . t_{n-1}-C_{n-1} \cdot t_{n}=0 \\
1 . t_{1}+1 . t_{2}+1 . t_{3}+\ldots+1 . t_{n-1}+1 . t_{n}=T,
\end{gathered}
$$

where $t_{i}$ is the time interval for the ith depth interval from the top of the section and $T$ is the total time interval from paleomagnetic and/or isotope analyses. The coefficients $C_{i}$ are derived from

$$
C_{n}=\frac{X_{n}}{\left(\frac{r_{n}}{r_{n+1}}\right)} \cdot x_{n+1},
$$

where $X_{n}$ is the $n t h$ depth interval counting from top down, and $r_{n}$ is the potassium to potassium plus calcium ratio as discussed above.

This equation set can be easily solved to yield a variable sedimentation rate throughout the section. In cases where the underlying pelagic sedimentation is not calcic then the elements involved in the ratio can be suitably modified. The variable stretching effect of using this modified sedimentation rate equation on the two sections is shown in Figure 6 as is the improved correlation of the potassium values in the two wells. Note especially the feature at $2.84 \mathrm{Ma}$.

The original data sequence (evenly sampled in the case of gammalog data, unevenly sampled in the case of core data) was resampled using linear interpolation at a sample interval that yielded a $2^{n}$ number of samples. The resampling at $1.3 \mathrm{k}$.y. gave 512 samples over a 666 k.y. time interval for all three data sets. If this sample interval is converted to depth, it results in an average sample interval of $3.6 \mathrm{~cm}$ for Hole $834 \mathrm{~A}$ and $10.5 \mathrm{~cm}$ for Hole $835 \mathrm{~A}$. In both these cases, the resample interval is less than the original sample interval and no information has been lost.

\section{Frequency Analysis}

Practical problems associated with using discrete fast Fourier transforms have been discussed by many people (Davis, 1973; Weedon, 1991; Priestley, 1981). The principal problems are concerned with sample frequency, number, and regularity, sharp ends to the sequence, and stationarity. The processing sequence was designed to minimize most of these effects.

A best-fit polynomial up to order 4 was first identified over the interval of interest. The sequence was detrended using this best-fit polynomial. This was considered more appropriate than a linear detrend. The long period cycles that are removed by this process could not be significantly resolved within this time/depth window.

The detrended data were standardized to yield a mean of zero and a variance of unity. The data were then resampled to give $512\left(2^{9}\right)$ samples. The resampling was achieved by linear interpolation. Both polynomial and "sinc" interpolations function well with low variance and equally spaced original data sequences such as wireline-log data. With high variance, irregularly spaced chemical analysis data, how- ever, both "sinc" and 3rd-order polynomial interpolations produced geologically unrealistic series. This is not saying, of course, that a linear interpolation yields a more realistic series; it is more an explicit statement of lack of knowledge about the true interpolation function.

Ten percent of the data at each end were tapered using a cosine taper (Weedon, 1991). The series was wrapped around (last data-point to first data-point) before tapering and a mid-point chosen such that the zero-end of the taper coincided with the least active part of the data window.

\section{Sequency Analyses}

Data preparation before conducting a Walsh transform is somewhat different. Because the amplitudes are not important in a Walsh transform, the data were recoded to +1 and -1 according to the following steps. The data were first detrended as for Fourier analysis. The detrended data were standardized to give a mean of zero and a variance of unity. The standardized sequence was then recoded by assigning +1 to all values over or equal to zero, and -1 to all other values.

Examples of original gamma data and the converted data before frequency and sequency analyses are shown in Figure 7.

\section{RESULTS}

The small number of ICAP data points in the interval of interest ( 57 in the case of Site 835, and 23 in the case of Site 834) means that neither frequency or sequency analyses produced significant results over the dated interval.

In the case of the wireline log data, although the regular sample intervals were adequate for the identification of cyclicity down to 10 k.y. (Site 835) and 28 k.y. (Site 834), no significant peaks were identified that could be unambiguously related to sediment cyclicity rather than aliasing and colored noise (Weedon, 1991). Further work will be necessary to evaluate the significance of the spectral results.

As could be expected, there is a difference between the spectra obtained using the linearly interpolated ages, and those based on the potassium/calcium ratio ages. In Figure 8 an apparent concentration of energy is seen in both wells between 25 and 30 k.y., 40-50 k.y., and 60-90 k.y. if we assume an average sedimentation rate over the time interval selected. If we assume that the relative potassium concentration reflects the sedimentation rate, then the two holes are still well correlated but the energy concentration has shifted to between 35 and 60 k.y.

\section{DISCUSSION}

Apart from presenting chemical analyses of cores that will provide a useful comparison with the geochemical logging data (Pratson et al. this volume) we have also shown how the core or cuttings geochemistry may have a role, together with paleomagnetic and isotope data, in suggesting possible objective stretching functions for converting depth to geological time. The improved correlation between Sites 834 and 835 that result from such a stretching function, particularly over the interval from 2.84 to $2.48 \mathrm{Ma}$ suggests that, over this interval at least, similar events were influencing both sites even though the volumes of sediment were different. This similarity of process is demonstrated by the spectral match between the two sites shown in Figure 8. Although the concentration of energy between 30 and 60 $\mathrm{k} . \mathrm{y}$. is very similar at both sites, we have not considered the presence of hiatuses in the sections and have no other, independent evidence that sediment has been removed by submarine erosion. We feel that it would be unwise to draw conclusions from these data concerning the sedimentation periodicities involved in this part of the Lau Basin without further evidence for continuity of the section.

While we have demonstrated similarities between the Fourier spectra from the two sections there is still uncertainty as to its cause. Are we looking at several interfering sedimentary cycles, or different 
点

Site 834
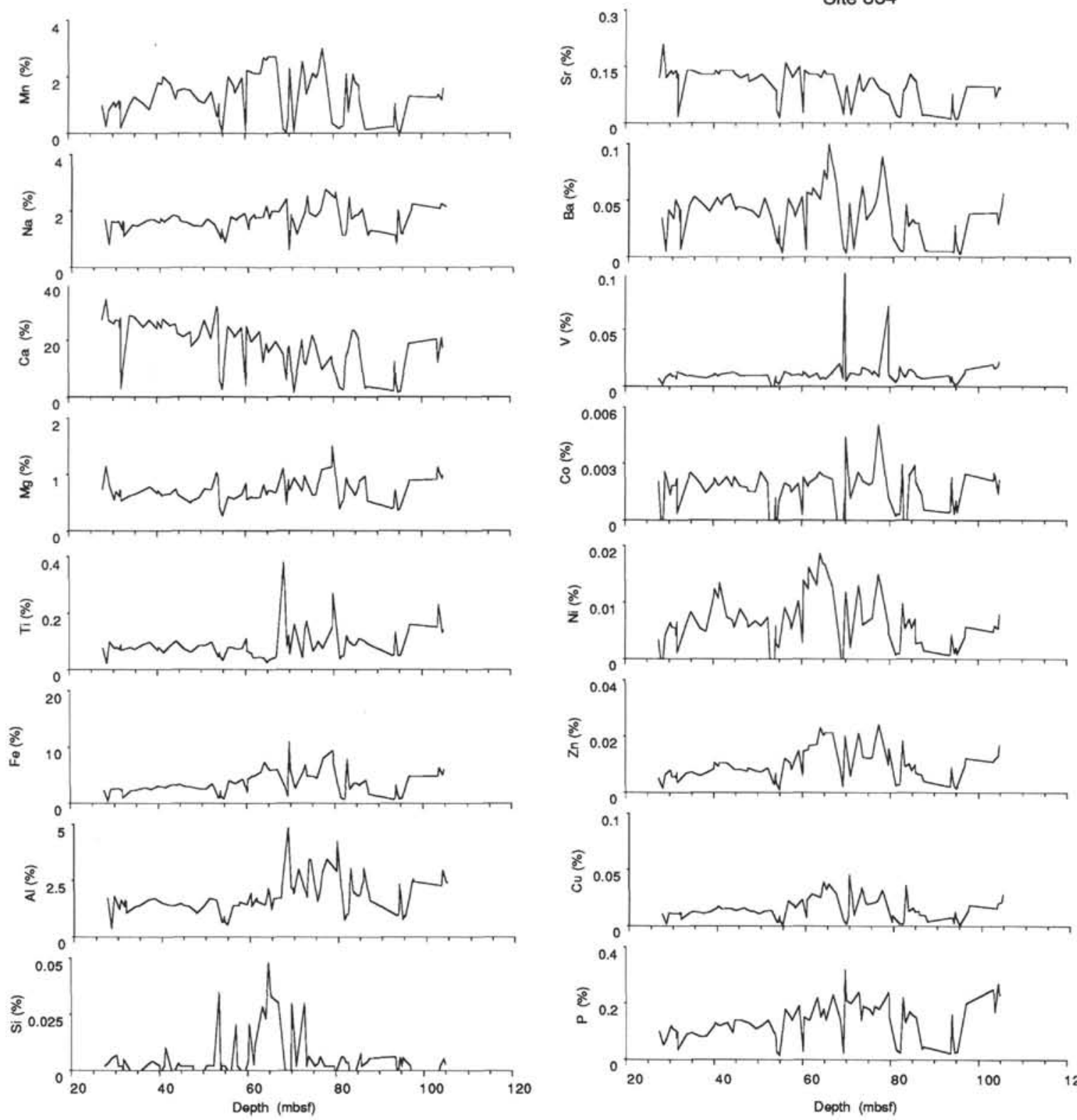
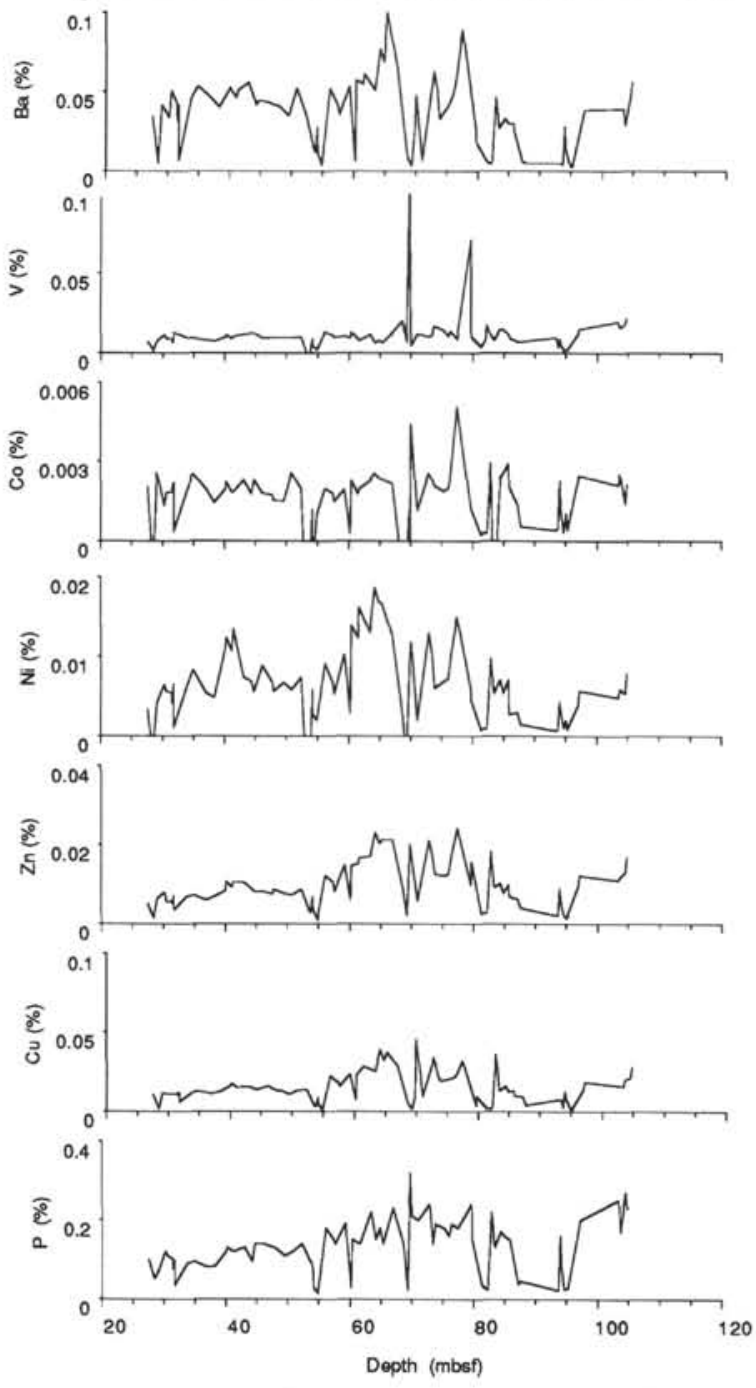
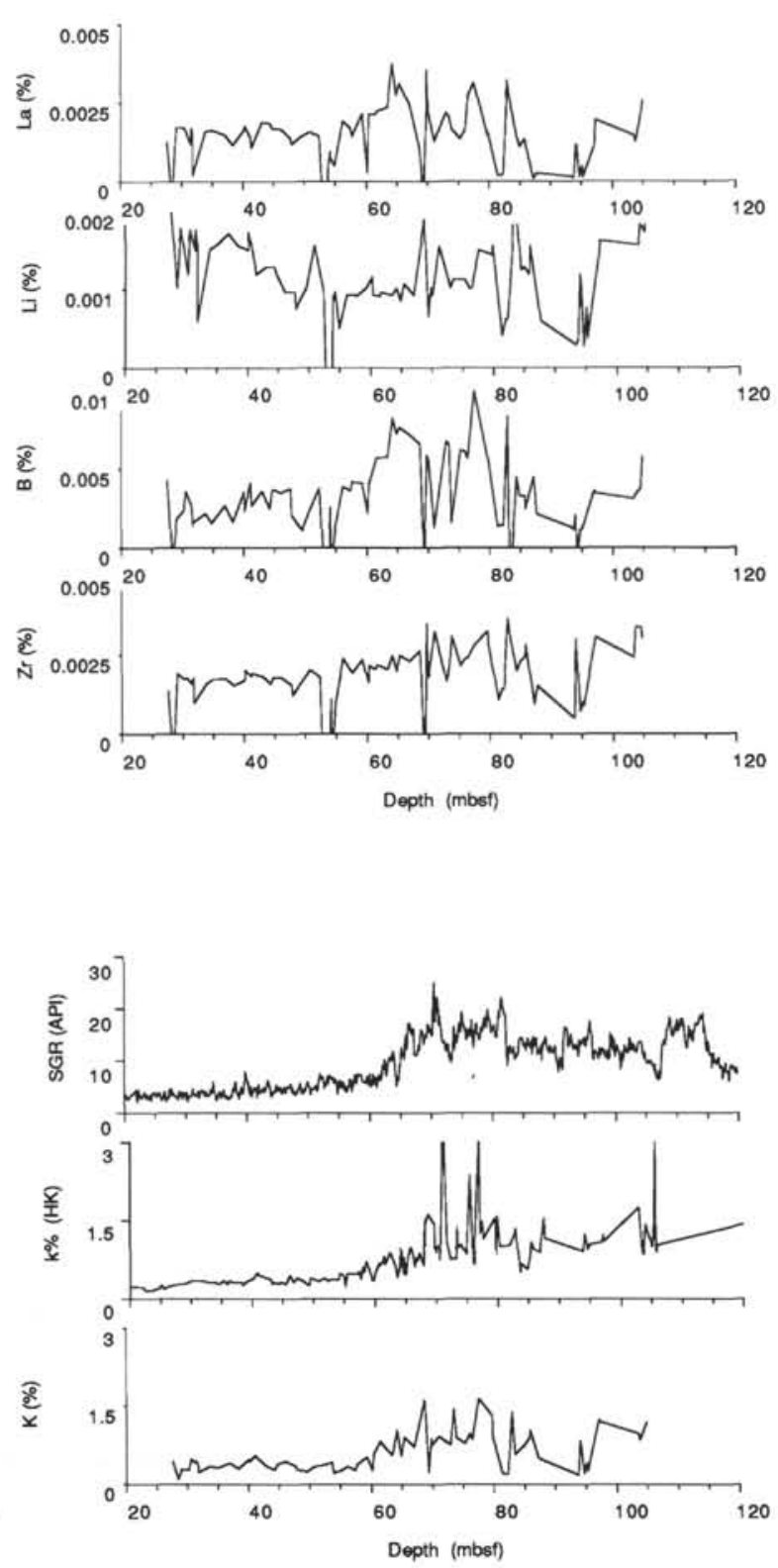

Figure 3. Plots of analytical results for Sites 834 and 835 . Element concentrations are in percent. Gamma-log response is in API units. 

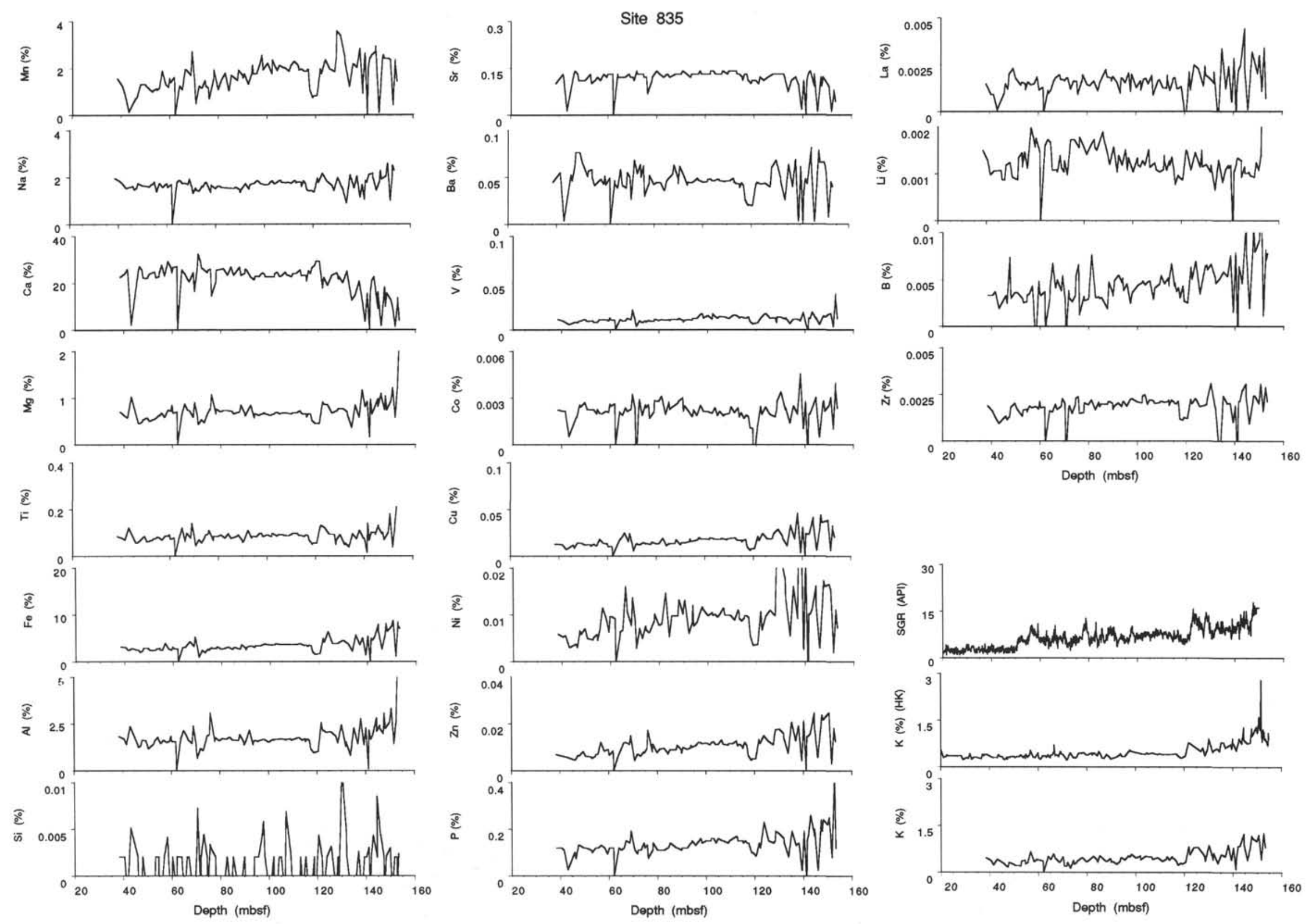


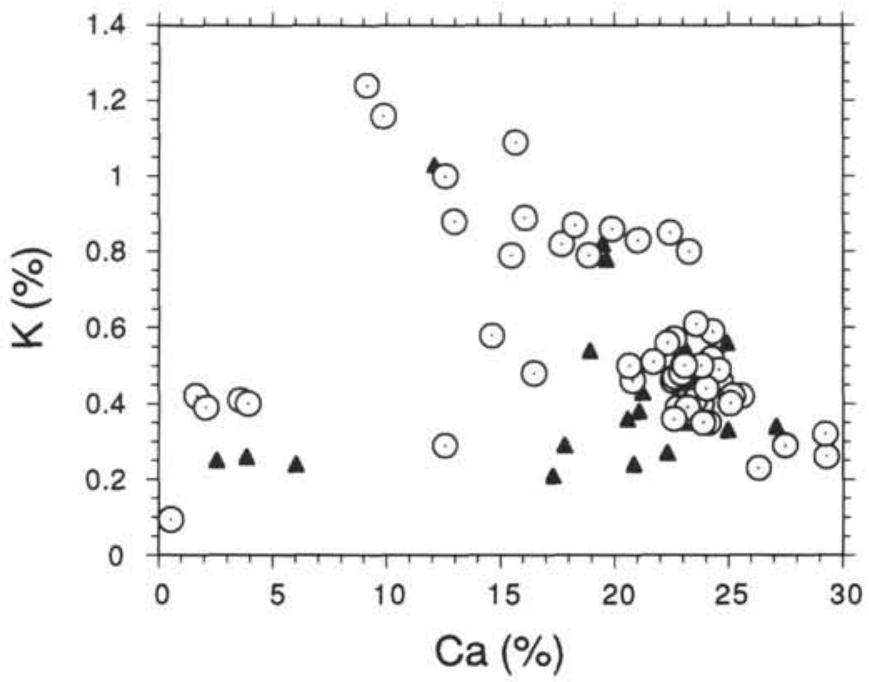

Figure 4. Plot of ICAP potassium vs. calcium, Holes $835 \mathrm{~A}$ (open circles) and $834 \mathrm{~A}$ (closed triangles).

A.



B.

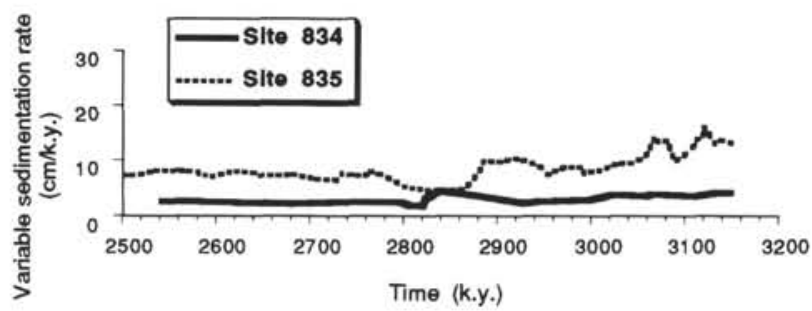

Figure 5. Sedimentation rates for Sites 834 and 835 . A. Relative sedimentation rates as a function of calcium, Holes $835 \mathrm{~A}$ (crosses) and 834A (closed triangles). B. Variable sedimentation rates at Sites 834 and 835 .

scales of events such as seismically promoted slumping or volcanic eruption frequency? Indeed, what is a cycle? One definition of a cycle would be an ordered passage through several states in sequence, the sequence being repeated through time. An on-off switch has only two states, with no intermediate positions. Can a series of on-off pulses be considered a cyclic sequence? A lighthouse with a single beam that pulses at a given frequency can be compared to a turbidite sequence that is switched on and off in response to earthquake tremors, with
A.

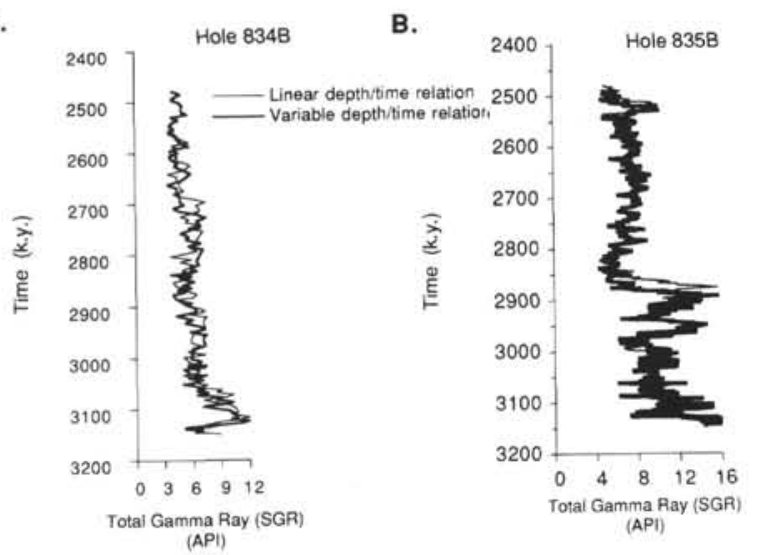

c.

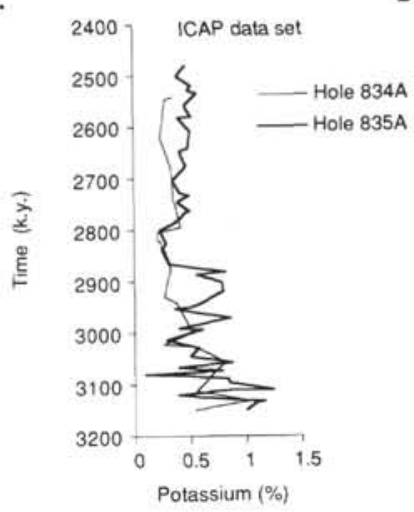

D.

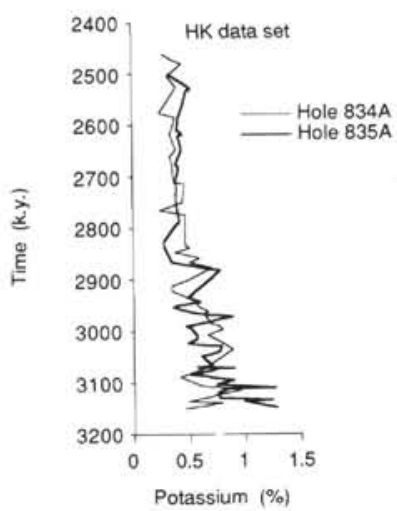

Figure 6. A, B. Wireline-log gamma values converted to geological time on the basis of both average sedimentation rate and the variable stretching approach discussed in the text. C. Correlation in geological time of the potassium values from ICAP analyses. The conversion to geological time is on the basis of the variable stretching approach discussed in the text for Holes $834 \mathrm{~A}$ and $835 \mathrm{~A}$. D. Correlation in geological time of the potassium values from the HK data set. The conversion to geological time is on the basis of the variable stretching approach discussed in the text for Holes 834A and 835A.

larger flows associated with larger earthquakes at longer intervals of time, or volcanic eruptions of varying size and quiescent periods. A spectral analysis of the lighthouse beam would give us the frequency/sequency of the signal without distinguishing between a true underlying sine/cosine function (for example, a Milankovitch forcing cycle) or a lighthouse keeper with a nervous disposition. In this sense the distinction between events and cycles becomes blurred. In a backarc basin the sedimentation may be expected to be dominated by tectonically driven pulses, and Walsh spectra may be expected to provide a more coherent picture of the event sequency. In the present case, however, the Fourier spectra give a clearer correlation. This seems likely to be caused by the relation between pelagic sedimentation and the turbidite pulses, giving a multistate cycle that consists of internal turbidite bundle structure and variations in pelagic mineralogy.

The prevalence of debris flows at Site 835 during the period from 2.9 to $2.1 \mathrm{Ma}$ (Rothwell et al., this volume), compared to the prevalence of hemipelagics at Site 834 during the same interval, will be reflected in the spectra only if the frequency of the causal mechanism is different (provided we work on a time rather than depth basis). Provided the hemipelagic sedimentation rate is similar at both sites, the thin turbidites at Site 834 and the thick debris flows at Site 835 can be expected to give similar spectra on a time base derived from sedimentation rates that vary as a function of lithology. This may explain the reasonable agreement in Fourier spectra for the two wells, 


\section{Hole 834B}


Hole 835B


Figure 7. The processing sequence for corrected natural gamma-ray data for Holes $834 \mathrm{~B}$ and $835 \mathrm{~B}$ before frequency and sequency analyses as discussed in the text. 
and the improved correlation based on the variable sedimentation rate even though the two sites have been subjected to different sedimentation histories.

However, a significant difference exists between sites over the lower part of the section, from 3.15 to $2.84 \mathrm{Ma}$. The correlation is not improved by using the objective stretching function. This either supports the hypothesis discussed by Rothwell et al. (this volume) that the lower part of Site 835 represents a single debris-flow deposit, or suggests that the $\mathrm{K}: \mathrm{Ca}$ ratio method of adjusting sedimentation rates is not applicable in this section.

On the other hand, it may be that the spectra are telling us that the debris flow period is, in reality, limited to the lower section from 3.15 to $2.84 \mathrm{Ma}$, and the younger section is in fact either dominated by hemipelagics at both sites, or both sites are subject to the same turbidite/debris flow influences.

At both sites the total gamma response (SGR) shows good agreement with both the ICAP and HK potassium core data, indicating that the influence of thorium and uranium is limited.

\section{ACKNOWLEDGMENTS}

The authors would like to thank Magne Ødegård for help with the chemical analysis. The financial support of the Norwegian Council for Science and Humanities (NAVF) is gratefully acknowledged. Financial support for R.A.H. was provided by the N.E.R.C., U.K. Constructive reviews by Graham Weedon and Gerhard Einsele were much appreciated.

\section{REFERENCES}

Davis, J.C., 1973. Statistics and Data Analysis in Geology: New York (Wiley). Golovchenko, X., Borella, P.E., and O'Connell, S., 1992. Sedimentary cycles on the Exmouth Plateau. In von Rad, U., Haq, B.U., et al., Proc. ODP, Sci. Results, 122: College Station, TX (Ocean Drilling Program), 279-291.

Harland, W.B., Armstrong, R.L., Cox, A.V., Craig, L.E., Smith, A.G., Smith, D.G., 1989. A Geologic Time Scale 1989. Cambridge (Cambridge Univ. Press).

Kanasewich, E.R., 1981. Time Sequence Analysis in Geophysics (3rd ed.): Edmonton, Alberta (Univ. of Alberta Press).

\section{Fourier}

A.

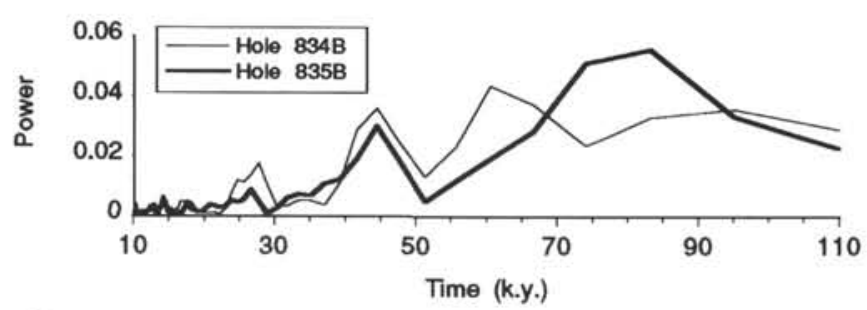

C.

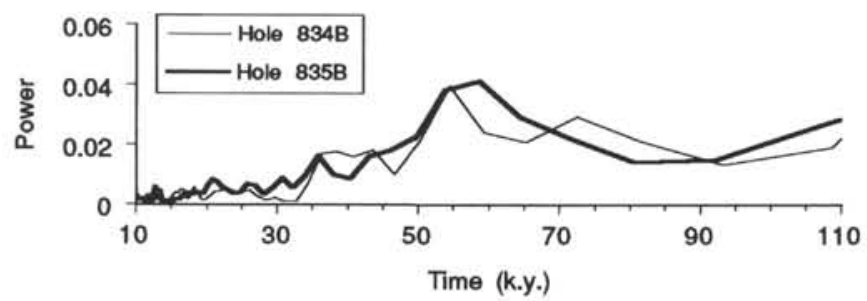

Karig, D.E., 1970. Ridges and basins of the Tonga-Kermadec island arc system. J. Geophys. Res., 75:239-254.

Malahoff, A., Feden, R.H., and Fleming, H.S., 1982. Magnetic anomalies and tectonic fabric of marginal basins north of New Zealand. J. Geophys. Res. $87: 4109-4125$.

Ødegård, M., 1981. The use of inductively coupled argon plasma (ICAP) atomic emission spectroscopy in the analysis of stream sediments. $J$. Geochem. Explor., 14:119-130.

, 1983. Utvidet program for analyse av geologiske materialer basert på syreekstraksjon og plasmaspektrometri. Geol. Surv. Norway, NGU Rep., 213.

Parson, L., Hawkins, J., Allan, J., et al., 1992. Proc. ODP, Init. Repts., 135: College Station, TX (Ocean Drilling Program).

Priestley, M.B., 1981. Spectral Analysis and Time Series: New York (Academic Press).

Schwarzacher, W., 1975. Sedimentation Models and Quantitative Stratigraphy: Amsterdam (Elsevier), Dev. in Sedimentol. Ser., 19.

1987. The analysis and interpretation of stratification cycles. $\mathrm{Pa}$ leoceanography, 2:79-95.

Smith, D.G., 1989. Milankovitch cyclicity and the stratigraphic record-a review. Terra Nova, 1:402-404.

Thompson, M., and Walsh, J.N., 1989. The Handbook of Inductively Coupled Plasma Spectrometry (2nd ed.): London (Blackie), 156-160.

Weedon, G.P., 1989. The detection and illustration of regular sedimentary cycles using Walsh power spectra and filtering, with examples from the Lias of Switzerland. J. Geol. Soc. London, 146:133-144.

, 1991. The spectral analysis of stratigraphic time series. In Einsele, G., Selacher, A., and Ricken, W. (Eds.), Cyclic and Event Stratification (2nd ed.): Berlin (Springer-Verlag), 840-855.

Worthington, P.F., 1990. Sediment cyclicity from well logs. In Hurst, A., Lovell, M.A., and Morton, A.C. (Eds.), Geological Applications of Wireline-logs. Geol. Soc. Spec. Publ. London, 48:123-132.

\footnotetext{
Abbreviations for names of organizations and publications in ODP reference lists follow the style given in Chemical Abstracts Service Source Index (published by American Chemical Society).
}

Date of initial receipt: 10 July 1992

Date of acceptance: 14 January 1993

Ms 135SR-105

B.

Walsh

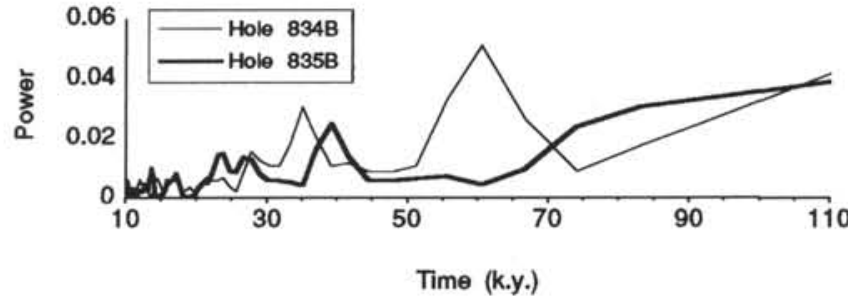

D.

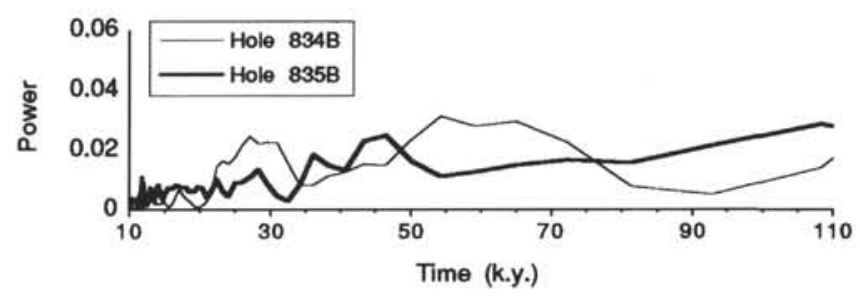

Figure 8. The resulting smoothed frequency and sequency spectra for Holes 834B and 835B. The spectra are derived from Fourier and Walsh analyses of the depth-to-time converted sections. A. Fourier power spectra for Holes $834 \mathrm{~B}$ and $835 \mathrm{~B}$, assuming a linear (average) sedimentation rate at each site. B. Walsh power spectra for Holes 834B and 835B, assuming a linear (average) sedimentation rate at each site. C. Fourier power spectra for Holes 834B and 835B, assuming a nonlinear sedimentation rate at each site. D. Walsh power spectra for Holes $834 \mathrm{~B}$ and $835 \mathrm{~B}$, assuming a nonlinear sedimentation rate at each site. 